\title{
ECONOMETRICA
}

JOURNAL OF THE ECONOMETRIC SOCIETY

An International Society for the Advancement of Economic Theory in its Relation to Statistics and Mathematics

http://www.econometricsociety.org/

Econometrica, Vol. 82, No. 1 (January, 2014), 89-135

PRICE SETTING WITH MENU COST FOR MULTIPRODUCT FIRMS

FERNANDO Alvarez

University of Chicago, Chicago, IL 60637, U.S.A.

FRANCESCO LIPPI

EIEF and University of Sassari, 00187 Rome, Italy

The copyright to this Article is held by the Econometric Society. It may be downloaded, printed and reproduced only for educational or research purposes, including use in course packs. No downloading or copying may be done for any commercial purpose without the explicit permission of the Econometric Society. For such commercial purposes contact the Office of the Econometric Society (contact information may be found at the website http://www.econometricsociety.org or in the back cover of Econometrica). This statement must be included on all copies of this Article that are made available electronically or in any other format. 


\title{
PRICE SETTING WITH MENU COST FOR MULTIPRODUCT FIRMS
}

\author{
By FERNANDO ALVAREZ AND FRANCESCO LIPPI ${ }^{1}$
}

\begin{abstract}
We model the decisions of a multiproduct firm that faces a fixed "menu" cost: once it is paid, the firm can adjust the price of all its products. We characterize analytically the steady state firm's decisions in terms of the structural parameters: the variability of the flexible prices, the curvature of the profit function, the size of the menu cost, and the number of products sold. We provide expressions for the steady state frequency of adjustment, the hazard rate of price adjustments, and the size distribution of price changes, all in terms of the structural parameters. We study analytically the impulse response of aggregate prices and output to a monetary shock. The size of the output response and its duration both increase with the number of products; they more than double as the number of products goes from 1 to 10 , quickly converging to the response of Taylor's staggered price model.
\end{abstract}

KEYWORDS: Menu cost, economies of scope in price changes, optimal control in multiple dimensions, fixed costs, monetary shocks, impulse responses.

\section{INTRODUCTION AND OVERVIEW}

THIS PAPER STUDIES A CLASSIC QUESTION IN MONETARY ECONOMICS, namely the impact of an unexpected monetary shock on prices and output in an economy where prices are sticky due to the presence of a fixed cost (a "menu" cost). We follow up on the program started by Golosov and Lucas (2007) and evaluate this impact by selecting, among menu cost models, those with the best ability to account for the patterns that are seen in the microeconomic data. To this end, we develop an analytically tractable model of the optimal pricesetting decisions of a firm that faces a fixed cost of simultaneously changing the prices of its $n \geq 1$ products. The hypothesis of a common cost for a simultaneous price change was proposed by Lach and Tsiddon $(1996,2007)$ as a way to generate small price changes, which have since been found in many data sets; see, for instance, Klenow and Malin (2010). A straightforward implication of this hypothesis is that the price changes of the firm's $n$ products are synchronized, a feature that has found empirical support in several papers. ${ }^{2}$

${ }^{1}$ We thank a co-editor and three anonymous referees. We benefited from discussions with Andy Abel, Ricardo Caballero, Carlos Carvalho, John Leahy, Bob Lucas, Virgiliu Midrigan, Luigi Paciello, Ricardo Reis, Raphael Schoenle, Kevin Sheedy, Rob Shimer, Paolo Surico, Nancy Stokey, Harald Uhlig, and Ivan Werning, as well as seminar participants at EIEF, the University of Chicago, NYU, Tinbergen Institute, ASSA 2012, Hong Kong University, the Federal Reserve Banks of Chicago, Minneapolis, New York, and Philadelphia, the Bank of Italy, the European Central Bank, the London Business School, the Bank of Norway, and the 2012 NBER Monetary Economics Meeting in New York for their comments. Alvarez thanks the ECB for the Wim Duisenberg fellowship. Lippi thanks the Italian Ministry of Education for sponsoring this project as part of PRIN 2010-11. Part of the research for this paper was sponsored by the ERC Advanced Grant 324008. Katka Borovickova and Philip Barrett provided excellent assistance.

${ }^{2}$ See the end of Section 4 for a discussion of the evidence and an interpretation of the multiproduct assumption in our model in light of the evidence. 
We solve the firm's decision problem, derive the steady state predictions for a cross section of firms, and study the response of the aggregate economy to a monetary shock. The challenges involved with modeling the propagation of monetary shocks in canonical menu cost problems have led many authors to resort to numerical methods. Our contribution is to present an approximate analytical solution to the general equilibrium of an economy where firms face a multidimensional and nonconvex control problem.

There are two sets of results. The first one concerns the model's cross-section predictions in a steady state. The model substantially improves the ability of state-of-the-art menu cost models to account for observed price-setting behavior. As documented by several empirical studies and summarized by Klenow and Malin (2010), the data display a large mass of small price changes: the size distribution of price changes appears bell-shaped. Existing menu cost models cannot account for this fact. We show that when $n=1$ or $n=2$, as in the models of Golosov and Lucas (2007) and Midrigan (2011), respectively, the size distribution of price changes is bimodal and U-shaped, featuring a minimal amount of small price changes. Our model produces a bell-shaped distribution provided $n \geq 6$, thus accounting for a robust feature of the data while retaining tractability (any $n$ can be studied). Simple expressions are derived to map the model's fundamental parameters (the size of the menu cost, the variance of the shocks, the demand elasticity, the number of products sold) into observable statistics such as the frequency of price adjustment $N_{a}$ and the standard deviation of price changes $\operatorname{Std}\left(\Delta p_{i}\right)$.

The second set of results concerns the analytical characterization of the response of the aggregate price level and of output to a monetary shock. This characterization extends the pioneering contributions of Caballero and Engel (1993, 2007) by going beyond their analysis of the impact effect, allowing for any number of goods $(n \geq 1)$ and justifying their simplifying assumption of using the steady state decision rules to analyze the transition dynamics. The last result gives a proof and an intuitive explanation for when the general equilibrium feedback on decision rules can be "neglected" in these models. ${ }^{3}$ The analytical results highlight two key determinants of the size and the duration of the impulse response functions (IRF) of output and prices to a once and for all monetary shock. The size and duration of the IRF depend, for a given number of products $n$, on the steady state frequency of price changes $N_{a}$ as well as on the steady state standard deviation of price changes, $\operatorname{Std}\left(\Delta p_{i}\right)$. For given values of these steady state statistics, the shape of the IRF depends only on $n$. Compared to the previous literature, which focused almost exclusively on the frequency of price changes as a proxy of aggregate stickiness, our analysis suggests that the dispersion of price changes is also an important determinant

\footnotetext{
${ }^{3}$ This simplifying assumption was used by Caballero and Engel (2007). Golosov and Lucas (2007) noticed in their quantitative analysis that decision rules were very close to the steady state rules.
} 
of the real effect of a monetary shock. We show that the flexibility of the aggregate price level is highest in the classic menu cost model with $n=1$, due the strong "selection effect" of price changes discussed by Golosov and Lucas (2007), namely that the first price changes after a shock are also the largest, so that the CPI response to the shock is fast. Absent selection the timing and magnitude of the price changes are uncorrelated. We show that for small monetary shocks, the selection effect weakens as $n$ increases and vanishes completely as $n \rightarrow \infty$. In this case, the price level and the output response to shocks is linear, as in Taylor's (1980) model, and the real effects of monetary policy are maximal, about two times those of a model where $n=1$. Our analysis thus provides an upper bound to the real effect of monetary shocks that is still smaller (about half) than predicted by a Calvo pricing mechanism. We also analyze the effect of different sizes of monetary shocks on output, a hallmark of menu cost models. The effect is small whether the shock is small or large, since in the latter case all firms change prices. We characterize the value of the monetary shock for which the cumulated effect on output (the area under the IRF) is maximized. Interestingly, for a given $n$, the monetary shock that maximizes the cumulative output effect is about one-half of $\operatorname{Std}\left(\Delta p_{i}\right)$. Moreover, the maximum value of the cumulated output effect is proportional to $\operatorname{Std}\left(\Delta p_{i}\right) / N_{a}$. For example in economies with large steady state price stickiness (namely with $N_{a}=1$ ), the maximum cumulated output effect ranges from $0.6 \%$ of annual output for $n=1$ to $1.4 \%$ for a large $n$.

The technical challenges in the analytical study of price-setting problems with menu cost have led researchers to consider simple environments. For instance, a quadratic (approximate) profit function was used in the seminal work of Barro (1972), Dixit (1991), Tsiddon (1993), Section 5 of Sheshinski and Weiss (1992), Caplin and Leahy (1997), and Chapter 12 of Stokey (2008), among others. Moreover the idiosyncratic shocks considered are stylized, for example, random walks with constant volatility, as used in Barro (1972), Tsiddon (1993), Gertler and Leahy (2008), and Danziger (1999), among others. Likewise, our analytical solution rests on carefully chosen approximations. In particular, our solution for the firm's decision problem uses a second order approximation of the profit function and assumes no drift in the price gaps. ${ }^{4}$ Moreover the impulse response functions are computed using the steady state decision rules, that is, ignoring the general equilibrium feedback effect. The paper discusses several extensions of the basic model, allowing for drift (e.g., in inflation or aggregate productivity) as well as correlated shocks among the different goods and showing that the model retains a great deal of tractability. ${ }^{5}$

\footnotetext{
${ }^{4}$ In Alvarez, Lippi, and Paciello (2011), we proved that the zero inflation assumption provides a good approximation to the true rules for inflation rates that are small relative to the variance of idiosyncratic shocks, an assumption that seems appropriate for developed economies (see Gagnon (2009), Alvarez, Gonzalez-Rozada, Neumeyer, and Beraja (2011) for related evidence).

${ }^{5}$ We thank Ricardo Caballero and, especially Carlos Carvalho, for suggesting a twodimensional state space representation that allowed a tractable analysis of the problem with drift.
} 
In Section 6, we show that the correlation among the shocks tends to reinforce the selection effect, so that the real effect of monetary policy becomes smaller as correlation increases. Moreover, we compare the accuracy of the analytical results vis à vis the results produced by models that feature an asymmetric profit function, the presence of drift, and account for the general equilibrium feedback on decision rules following the aggregate shock. These investigations, reported in Appendix C in the Supplemental Material (Alvarez and Lippi (2014)), show that the approximate results obtained in the paper provide accurate predictions of the exact numerical solution produced by those more involved models for small (realistic) monetary shocks.

\section{Overview of the Analysis and Main Findings}

In Section 2, we set up the problem of a multiproduct firm that can revise prices only after paying a fixed cost. The key assumption is that once the fixed menu $\operatorname{cost} \psi$ is paid, the firm can adjust the price of all its products. We assume that the static profit maximizing prices for each of the $n$ products, that is, the prices that would be charged absent menu cost, follow $n$ independent random walks without drift and with volatility $\sigma$. We refer to the difference between the frictionless and the actual prices as to the (vector of) price gaps. The period return function is shown to be proportional to the sum of the squared price gaps. The proportionality constant $B$ measures the second order losses associated with charging a price different from the optimum, that is, it is a measure of the curvature of the profit function. The firm minimizes the expected discounted cost, which includes the stream of lost profits from charging prices different from the frictionless case, as well as the fixed cost at the time of the adjustments.

The solution of the firm's problem in Section 3 involves finding the set over which prices are adjusted and its complement, the "inaction" set. To our knowledge, this is the first fixed cost adjustment problem in $n$ dimensions whose solution is analytically characterized. Somewhat surprisingly, the solution to this complex problem turns out to have a simple form: the optimal decision is to control the price gaps so as to remain in the interior of the $n$-dimensional ball centered at the origin. The economics of this is clear: the firm will adjust either if many of its price gaps have a medium size or if a few gaps are very large. The size of this ball, whose squared radius is denoted by $\bar{y}$, is chosen optimally. We solve for the value function and completely characterize the size of the inaction set $\bar{y}$ as a function of the parameters of the problem. We show that the approximate solution $\bar{y} \approx\left[2(n+2) \sigma^{2} B / \psi\right]^{1 / 2}$ gives an accurate approximation of the exact solution for a small cost $\psi .^{6}$

In Section 4, we explore several steady state implications of the model. First we show that the expected number of price adjustments per unit of time, de-

\footnotetext{
${ }^{6}$ The special case of $n=1$ gives the same quartic root as in Barro (1972), Karlin and Taylor (1981), and Dixit (1991), since $\bar{y}$ is the square of the price threshold.
} 
noted by $N_{a}$, is given by $n \sigma^{2} / \bar{y}$, which, together with our result for $\bar{y}$, gives a complete characterization of the frequency of price adjustments. Second, we solve in closed form for the hazard rate of the price changes as a function of the time elapsed since the last change. The scale of the hazard function is determined by the expected number of adjustments $N_{a}$. Fixing the scale, the shape of this function depends exclusively on the number of products $n$. We show that the hazard rate gets steeper as $n$ increases. Third, while price changes occur simultaneously for the $n$ products, we characterize the marginal distribution of price changes, that is, the statistic that is usually computed in actual data sets. A closed form expression for the density of the marginal distribution of price changes as a function of $\bar{y}$ and $n$ is derived and used to compute several statistics, such as the standard deviation of price changes $\operatorname{Std}\left(\Delta p_{i}\right)$, and other moments that are only functions of $n$, such as the coefficient of variation and the excess kurtosis of the absolute value of price changes. As the number of products increases, the size of the adjustments decreases monotonically, that is, with more products, the typical price adjustment of each product is smaller. These cross-section predictions could be used to identify the parameters of the model and test its implications. We show that once the scale of price changes is controlled for, the shape of the size distribution is exclusively a function of the number of products $n$. For $n=2$, the distribution is bimodal, with modes at the absolute value of $\sqrt{\bar{y}}$; for $n=3$, it is uniform; for $n=4$, it peaks at zero and is concave; and for $n \geq 6$, it is bell-shaped. As $n \rightarrow \infty$, the density of price changes converges to a normal distribution.

In Section 5, we use the firm's optimal decisions to characterize the responses of the aggregate price level and output to a monetary shock. In doing this, we keep the decision rules of the firms constant, an approximation used in some of the calculations by Golosov and Lucas (2007) and Caballero and Engel (1991, 1993, 2007), among many others. Indeed, we justify this practice by proving that the general equilibrium feedback effects have negligible consequences on the size of the inaction region-a finding related to the result in Gertler and Leahy (2008). This result allows us to characterize analytically the effect on aggregate prices and on output of a permanent unexpected increase in money supply in an economy that starts at the cross-sectional stationary distribution of price gaps under zero inflation.

The analytical IRF of prices to a monetary shock is made of two pieces: an impact effect (a jump in the price level) and the remaining part. The IRF depends only on three parameters: the number of products, $n$, the frequency of price changes, $N_{a}$, and the standard deviation of price changes, $\operatorname{Std}\left(\Delta p_{i}\right)$. More precisely, the IRF is homogenous of degree 1 in the size of the shock, $\delta$, and in $\operatorname{Std}\left(\Delta p_{i}\right)$. Moreover, the duration of the impulse response is inversely proportional to the steady state frequency of price changes, $N_{a}$, that is, time can be measured relative to the steady state average duration of prices. When monetary shocks are larger than twice $\operatorname{Std}\left(\Delta p_{i}\right)$, the economy features complete price flexibility. In contrast, for small monetary shocks, the impact effect 
on prices is second order compared to the shock size and, hence, the impact effect on output is on the order of the monetary shock. These results, together with the homogeneity, characterize the precise sense in which the size of the shocks matters. Fixing the two steady state parameters, $N_{a}$ and $\operatorname{Std}\left(\Delta p_{i}\right)$, the whole shape of the impulse response depends only on the number of products $n$ and the normalized size of the shock $\delta / \operatorname{Std}\left(\Delta p_{i}\right)$. As we move from $n=1$ to a large number of products (say $n \geq 10$ ), the impact effect on prices, as well as the half-life of a monetary shock, more than double. Indeed, as $n \rightarrow \infty$, the impulse response converges to the response of Taylor's (1980) model or the inattentiveness model of Caballero (1989), Bonomo and Carvalho (2004), and Reis (2006). In this case, there is absolutely no selection and the impulse response is linear in time, and has-for small shocks-a half-life of $1 /\left(2 N_{a}\right)$, that is, half the average duration of steady state price changes. In the language of Golosov and Lucas (2007), economies with higher values of $n$ have a smaller amount of selection.

Our analysis extends Midrigan's (2011) contribution to any number of goods $n$, and derives the implication for the shape of the distribution of price changes and hazard rates that is not derived in his paper. We show that the $n=2$ case produces a size distribution of price changes that is "strongly" bimodal, very similar to the distribution in Golosov and Lucas. We show that a larger number of goods, on the order of $n=10$, is necessary to replicate qualitatively the large mass of small price changes and the bell-shaped distribution of price changes that are seen in the data. Concerning the hazard rate, Midrigan (2011, p. 1167) commented that "[e]conomies of scope flatten the adjustment hazard and thus weaken the strength of the selection effect even further." We show that without fat-tailed shocks, the hazard rate steepens with $n$ and, indeed, the economy converges to Taylor's staggered adjustment model, not to Calvo's (flat hazard) random adjustment model. Concerning the optimal decision rule for adjustment after an aggregate shock, Midrigan (2011, Section 4.B, pp. 11651168 ) interpreted the price adjustment decision in his model with $n=2$ using the techniques developed by Caballero and Engel for the case of $n=1$. We show in Section 5 that for the multiproduct case $(n>1)$, the threshold condition for price adjustments involves a vector of price gaps, not just one. Finally, we clarify how the "multiproduct hypothesis" affects the consequences of monetary shocks in comparison to the seminal paper of Golosov and Lucas (2007). Midrigan (2011) tackled this question numerically in a model where $n=2$ which, moreover, assumed the presence of infrequent large shocks. In Midrigan's paper the real effects of monetary shocks are about 4 times larger than in Golosov and Lucas. Our model shows that only a small part of this difference stems from the multiproduct hypothesis; the bulk of the difference is due to the infrequent large shocks. Our analytical results show that without the infrequent large shocks, the multiproduct hypothesis for the $n=2$ case produces real effects that are only $20 \%$ larger than in Golosov and Lucas (2007), and even smaller for the case of correlated shocks. We infer that the large effects of monetary shocks obtained by Midrigan are due to the presence of 
the infrequent large shocks, which reintroduce an element of stochastic time dependence (à la Calvo) in the price-setting decision.

\section{THE FIRM'S PROBLEM: SETUP AND INTERPRETATION}

Let $n$ be the number of products sold by the firm. The mathematical model we use has an $n$-dimensional state $p$ that we refer to as the vector of price gaps, whose interpretation is discussed below. Each price gap $p_{i}$, when it is not controlled, evolves according to a random walk without drift, so that $\mathrm{d} p_{i}=$ $\sigma \mathrm{d} \mathcal{W}_{i}$, where $\mathrm{d} \mathcal{W}_{i}$ is a standard Brownian motion and $\sigma$ the volatility. The $n$ Brownian motions (BM henceforth) are independent, so $\mathbb{E}\left[\mathcal{W}_{i}(t) \mathcal{W}_{j}\left(t^{\prime}\right)\right]=0$ for all $t, t^{\prime} \geq 0$ and $i, j=1, \ldots, n$. The value function $V(p)$ is the minimum value of the function $\mathbf{V}$ defined over the processes $\{\boldsymbol{\tau}, \Delta \mathbf{p}\} \equiv\left\{\tau_{j}, \Delta p_{i}\left(\tau_{j}\right)\right\}_{j=1}^{\infty}$,

$$
\begin{aligned}
V(p) & =\min _{\boldsymbol{\tau}, \mathbf{\Delta} \mathbf{p}} \mathbf{V}(\boldsymbol{\tau}, \mathbf{\Delta} \mathbf{p} ; p) \\
& \equiv \mathbb{E}\left[\sum_{j=1}^{\infty} e^{-r \tau_{j}} \psi+\int_{0}^{\infty} e^{-r t} B\left(\sum_{i=1}^{n} p_{i}^{2}(t)\right) d t \mid p(0)=p\right],
\end{aligned}
$$

where $r$ is the intertemporal discount rate, $B$ a parameter whose interpretation is discussed below, and each element of the vector of price gaps $p$ follows

$$
p_{i}(t)=\sigma \mathcal{W}_{i}(t)+\sum_{j: \tau_{j}<t} \Delta p_{i}\left(\tau_{j}\right) \quad \text { for all } t \geq 0 \text { and } i=1,2, \ldots, n
$$

$\Delta p_{i}\left(\tau_{j}\right) \equiv \lim _{t \downarrow \tau_{j}} p_{i}(t)-\lim _{t \uparrow \tau_{j}} p_{i}(t)$, and $p(0)=p$.

The $\tau_{j}$ are the (stopping) times at which control is exercised. At these times, after paying the cost $\psi$, the state can be changed to any value in $\mathbb{R}^{n}$. We denote the vector of changes in the price gaps as $\Delta p\left(\tau_{j}\right) \in \mathbb{R}^{n}$. This is a standard adjustment cost problem subject to a fixed cost, with the exception that after paying the adjustment cost $\psi$, the decision maker can adjust the state in the $n$ dimensions.

Next we discuss three interpretations of the problem that can be summarized by saying that the firm "tracks" the prices that maximize instantaneous profits from the $n$ products. In each interpretation, a monopolist sells $n$ goods with additively separable demands: first subject to costs shocks and, second, subject to demand shocks. For the first interpretation, consider a system of $n$ independent demands, with constant elasticity $\eta$ for each product, random multiplicative shifts in each of the demands, and a time varying marginal (and average) cost $W Z_{i}(t)$. This is a stylized version of the problem introduced by Midrigan (2011) where the elasticity of substitution between the products sold within the firm is the same as the elasticity of the bundle of goods sold across firms. The instantaneous profit maximizing price is proportional to the marginal cost; 
in logs, it is $p_{i}^{*}(t)=\log W+\log Z_{i}(t)+\log (\eta /(\eta-1))$. In this case, we assume that the log of the marginal cost evolves as a random walk with drift so that $p_{i}^{*}(t)$ inherits this property. The period cost is a second order expansion of the profit function with respect to the vector of the log of prices, around the prices that maximize current profits (see Section 5 and Appendix B in the Supplemental Material for a detailed presentation of this interpretation). The units of the objective function are (lost) profits normalized by the maximum profits of producing one good. The first order price-gap terms in the expansion are zero because we are expanding around $p^{*}(t)$. There are no second order cross-terms due to the separability of the demands. Thus we can write the problem in terms of the gap between the actual price and the profit maximizing price: $p(t)=\hat{p}(t)-p^{*}(t)$. Under this approximation, $B$ is given by $B=(1 / 2) \eta(\eta-1)$. Likewise, the fixed cost $\psi$ is measured relative to the maximum profits of producing one good. Clearly all that matters to characterize the decision rules is the ratio of $B$ to $\psi$, for which purpose the units in which we measure them is immaterial. For the second interpretation of the model, consider a monopolist facing identical demands for each of the $n$ products that she sells. The demands are linear in their own price and have zero crosspartials with respect to the other prices. The marginal costs of producing each of the products are also identical and assumed to be linear. The intercepts of each of the $n$ demands follow independent standard BM's. In this interpretation, the firm's profits are the sum of the $n$ profit functions derived in the seminal work by Barro (1972), so that our $\psi$ is his $\gamma$ and our $B$ is his $\theta$, as defined in his equation (12). A third interpretation is in terms of an optimal inattention or inattentiveness problem, similar to that studied by Reis (2006), and Alvarez, Lippi, and Paciello (2011). The firm has the same demand system for the $n$ products and, hence, the same total period losses $B\|p(t)\|^{2}$, which are assumed to be continuously and freely observed. Furthermore, if the firm pays an observation cost $\psi$, it observes the determinants of the profits of each of the products separately and is able to set prices based on this information. In this case, $\psi$ represents the cost of gathering and processing the information, in addition to (or instead of) the menu cost of changing prices.

\section{CHARACTERIZATION OF THE FIRM'S DECISIONS}

We first note the following properties of the firm's problem:

P1. Given the symmetry of the return function, of the law of motion, and of target prices, it is immediate that after an adjustment, the state is reset at the origin, that is, $p\left(\tau_{j}^{+}\right)=0$ or $\Delta p\left(\tau_{j}\right)=-p\left(\tau_{j}^{-}\right)$.

P2. The state space $\mathbb{R}^{n}$ can be divided into two open sets: an inaction region $\mathcal{I} \subset \mathbb{R}^{n}$ and a control region $\mathcal{C} \subset \mathbb{R}^{n}$. We use $\partial \mathcal{I}$ for the boundary of the inaction region. We have that $\mathcal{C} \cap \mathcal{I}=\emptyset$, that inaction is strictly preferred in $\mathcal{I}$, that control is strictly preferred in $\mathcal{C}$, and that in $\partial \mathcal{I}$ the agent is indifferent between control and inaction. 
P3. The instantaneous return of the problem in equation (1) is a function of the scalar $y$, the squared norm of the vector of price gaps:

$$
y=\sum_{i=1}^{n} p_{i}^{2} \geq 0 .
$$

P4. The process for $y$ is a one-dimensional diffusion given by

$$
\mathrm{d} y=n \sigma^{2} \mathrm{~d} t+2 \sigma \sqrt{y} \mathrm{~d} \mathcal{W} \text { for } y \in[0, \bar{y}] .
$$

To better understand, use Ito's lemma on equation (3) to get $\mathrm{d} y=n \sigma^{2} \mathrm{~d} t+$ $2 \sigma \sum_{i=1}^{n} p_{i}(t) \mathrm{d} \mathcal{W}_{i}$, implying $\mathbb{E}(\mathrm{d} y)^{2}=4 \sigma^{2}\left(\sum_{i=1}^{n} p_{i}^{2}(t)\right) \mathrm{d} t$, which gives the diffusion shown above.

P5. Points P3 and P4 imply that the $n$-dimensional state of the original problem and decision rules can be summarized by a single scalar, namely $y$. The optimal policy for this problem is given by a threshold rule such that if $y<\bar{y}$, there is inaction. The first time that $y$ reaches $\bar{y}$, all prices are adjusted to the origin, so that $y=0$. The one-dimensional problem has the value function

$$
v(y)=\min _{\bar{y}} \mathbb{E}\left[\sum_{j=1}^{\infty} e^{-r \tau_{j}} \psi+\int_{0}^{\infty} e^{-r t} B y(t) d t \mid y(0)=y\right]
$$

subject to equation (4) for $y \in[0, \bar{y}]$ and the $\tau_{j}$ 's are the times at which $y(t)$ hits $\bar{y}$.

The function $v$ solves

$$
r v(y)=B y+n \sigma^{2} v^{\prime}(y)+2 \sigma^{2} y v^{\prime \prime}(y) \text { for } y \in(0, \bar{y}) .
$$

Since policy calls for adjustment at values higher than $\bar{y}$, we have $v(y)=$ $v(0)+\psi$ for all $y \geq \bar{y}$. If $v$ is differentiable at $\bar{y}$, we can write the two boundary conditions

$$
v(\bar{y})=v(0)+\psi \quad \text { and } \quad v^{\prime}(\bar{y})=0 .
$$

These conditions are typically referred to as value matching and smooth pasting. For $y=0$ to be the optimal return point, it must be a global minimum, and thus we require that $v^{\prime}(0) \geq 0$. Note the weak inequality, since $y$ is nonnegative.

The next proposition gives an analytical solution for $v$ in the range of inaction.

Proposition 1: Let $\sigma>0$. The ordinary differential equation $(O D E)$ given by equation (6) is solved by the analytical function

$$
v(y)=\sum_{i=0}^{\infty} \beta_{i} y^{i} \quad \text { for } \quad y \in[0, \bar{y}]
$$


where for any $\beta_{0}$, the coefficients $\left\{\beta_{i}\right\}_{i=1}^{\infty}$ solve

$$
\begin{aligned}
& \beta_{0}=\frac{n \sigma^{2}}{r} \beta_{1}, \quad \beta_{2}=\frac{r \beta_{1}-B}{2 \sigma^{2}(n+2)}, \\
& \beta_{i+1}=\frac{r}{(i+1) \sigma^{2}(n+2 i)} \beta_{i} \quad \text { for } \quad i \geq 2 .
\end{aligned}
$$

The next proposition shows that there exists a unique solution of the ODE (6) that satisfies the relevant boundary conditions (see Appendix A for the proofs).

Proposition 2: Assume $r>0, \sigma>0$, and $n \geq 1$. There exist $\bar{y}$ and a unique function $v(\cdot)$ solving the ODE (6) that satisfy the boundary conditions in equations (7). Moreover, (i) $v(y)$ is minimized at $y=0$, (ii) $v(y)$ is strictly increasing in $(0, \bar{y})$, and (iii) $\bar{y}$ is a local maximum, that is, $\lim _{y \uparrow \bar{y}} v^{\prime \prime}(y)<0$.

We note that a slightly modified version of a verification theorem in $\varnothing$ ksendal (2000) can be used to prove that value function $v$ and threshold policy $\bar{y}$ that we found in Proposition 2 for the one-dimensional representation indeed characterize the inaction $\mathcal{I}=\left\{p:\|p\|^{2}<\bar{y}\right\}$ and control sets $\mathcal{C}$, as well as the value function $V(p)$ for the original $n$-dimensional problem (see Appendix C in Alvarez and Lippi (2012) for more details and references to related results in the applied math literature). We finish this section by characterizing the optimal policy $\bar{y}$ in terms of the structural parameters of the model $\left(\frac{\psi}{B}, \sigma^{2}, n, r\right)$.

PROPOSITION 3: The optimal threshold is given by a function $\bar{y}=\frac{\sigma^{2}}{r} Q\left(\frac{\psi r^{2}}{B \sigma^{2}}, n\right)$ so that

(i) $\bar{y}$ is strictly increasing in $\frac{\psi}{B}$ with $\bar{y}=0$ if $\frac{\psi}{B}=0$ and $\bar{y} \rightarrow \infty$ as $\frac{\psi}{B} \rightarrow \infty$,

(ii) $\bar{y}$ is strictly increasing in $n$ and $\bar{y} \rightarrow \infty$ as $n \rightarrow \infty$,

(iii) $\bar{y}$ is bounded below by $\sqrt{2(n+2) \sigma^{2} \frac{\psi}{B}}$ and as $\frac{\psi}{B} \frac{r^{2}}{\sigma^{2}} \rightarrow 0$, then $\frac{\bar{y}}{\sqrt{2(n+2) \sigma^{2} \psi / B}} \rightarrow 1$,

(iv) the elasticity of $\bar{y}$ with respect to $r$ and $\sigma^{2}$ satisfies

$$
\frac{r}{\bar{y}} \frac{\partial \bar{y}}{\partial r}=2 \frac{(\psi / B)}{\bar{y}} \frac{\partial \bar{y}}{\partial(\psi / B)}-1 \quad \text { and } \quad \frac{\sigma^{2}}{\bar{y}} \frac{\partial \bar{y}}{\partial \sigma^{2}}=1-\frac{(\psi / B)}{\bar{y}} \frac{\partial \bar{y}}{\partial(\psi / B)} .
$$

See Appendix A for the proof. When $n=1$, the formula for the threshold is the same expression derived by Barro (1972), Karlin and Taylor (1981), and Dixit (1991), though our characterization is a bit more general and, more im- 
portantly, holds for any number of products $n \geq 1 .^{7}$ That $\bar{y}$ is only a function of the ratio $\psi / B$ is apparent from the definition of the sequence problem. That, as stated in part (i), $\bar{y}$ is strictly increasing in the ratio of the fixed cost to the benefit of adjustment $\psi / B$ is quite intuitive. Item (ii) says that the threshold is increasing in the number of products $n$. This is because as $n$ increases, equation (4) shows that the drift of $y=\|p\|^{2}$ increases; thus if $\bar{y}$ is held constant, there will be more adjustments per unit of time and hence higher menu costs will be paid. Additionally, if $\bar{y}$ remains unchanged, the average cost per unit of time also increases. One can show that the second effect is smaller and, hence, an increase in $n$ makes it optimal to increase $\bar{y}$. Part (iii) gives an expression for a lower bound for $\bar{y}$, which becomes arbitrarily accurate for either a small value of the cost $\psi / B$, so that the range of inaction is small, or a small value of the interest rate $r$, so that the problem is equivalent to minimizing the steady state average net cost. We note that in the approximation

$$
\bar{y}=\sqrt{\frac{\psi \sigma^{2} 2(n+2)}{B}},
$$

the effect of $\psi \sigma^{2} / B$ is exactly the same as in the case of one product. Note that the approximation in part (iii) implies that the elasticity of $\bar{y}$ with respect to $\psi / B$ is $1 / 2$ for small values of the $\psi / B$ ratio. Then, using part (iv), we obtain that $\bar{y}$ has elasticity $1 / 2$ with respect to $\sigma^{2}$ and also that it is independent of $r$. Moreover, for small normalized adjustment cost, that is, as $\psi /\left(B \sigma^{2}\right) \downarrow 0$, (iii) and (iv) imply that $\partial \bar{y} / \partial r \rightarrow 0$, so that interest rates have only second order effects on the range of inaction. Finally, we found that the quadratic approximation to $v(\cdot)$, which amounts to a quartic approximation to $V(\cdot)$, gives very accurate values for $\bar{y}$ across a very large range of parameters. What happens is that for a realistic application, the values of $r$ and $\psi$ are small relative to $B \sigma^{2}$, hence the approximation of part (iii)) applies. ${ }^{8}$

\section{IMPLICATIONS FOR FREQUENCY AND SIZE OF PRICE CHANGES}

In this section, we explore the implications for the frequency and distribution of price changes. The expected time for $y(t)$ to hit the barrier $\bar{y}$ starting at $y$ is given by the function $\mathcal{T}(y)$ that satisfies $0=1+n \sigma^{2} \mathcal{T}^{\prime}(y)+2 y \sigma^{2} \mathcal{T}^{\prime \prime}(y)$ for $y \in(0, \bar{y})$ with a boundary condition $\mathcal{T}(\bar{y})=0$, which gives $\mathcal{T}(y)=\frac{\bar{y}-y}{n \sigma^{2}}$ for $y \in$ $[0, \bar{y}]$. Thus $\mathcal{T}(0)$ gives the expected time between successive price adjustments, so that the average number of adjustments, denoted by $N_{a}$, is $\frac{1}{\mathcal{T}(0)}$. We summarize this result in the following proposition.

\footnotetext{
${ }^{7}$ See expression (19) in Barro (1972) and Chapter 15, Section 3.F of Karlin and Taylor (1981) for the case of undiscounted returns; see expression (11) in Dixit (1991) for an approximation to the threshold for the discounted case.

${ }^{8}$ See Appendix D of Alvarez and Lippi (2012) for a numerical illustration.
} 
PROPOSITION 4: Let $N_{a}$ be the expected number of price changes for a multiproduct firm with $n$ goods. It is given by

$$
N_{a}=\frac{n \sigma^{2}}{\bar{y}}=\frac{n r}{Q\left(\frac{\psi r^{2}}{B \sigma^{2}}, n\right)} \cong \sqrt{\frac{B \sigma^{2}}{2 \psi} \frac{n^{2}}{(n+2)}}
$$

The second equality in equation (11) uses the function $Q(\cdot)$ derived in Proposition 3, while in the last equality, we use the approximation of $\bar{y}$ for small $\psi r^{2} /\left(B \sigma^{2}\right)$. It is interesting that this expression extends the well known expression for the case of $n=1$ simply by adjusting the value of the variance from $\sigma^{2}$ to $n \sigma^{2}$. The number of products $n$ affects $N_{a}$ through two opposing forces. On one hand, with more products, the variance of the deviations of the price gaps increases and, thus, a given value of $\bar{y}$ is hit sooner in expected value. This is the "direct effect." On the other hand, with more products, the optimal value of $\bar{y}$ is higher. Equation (11) shows that the direct effect dominates and the frequency of adjustment increases with $n$.

Next we characterize the hazard rate of price adjustments (see Appendix A for the proof).

PROPOSITION 5: Let $t$ denote the time elapsed since the last price change. Let $J_{\nu}(\cdot)$ be the Bessel function of the first kind. The hazard rate for price changes is given by

$$
\begin{aligned}
& h(t)=\sum_{k=1}^{\infty} \frac{\xi_{n, k}}{\sum_{s=1}^{\infty} \xi_{n, s} \exp \left(-\frac{q_{n, s}^{2} \sigma^{2}}{2 \bar{y}} t\right)} \frac{q_{n, k}^{2} \sigma^{2}}{2 \bar{y}} \exp \left(-\frac{q_{n, k}^{2} \sigma^{2}}{2 \bar{y}} t\right), \\
& \text { where } \quad \nu=\frac{n}{2}-1, \\
& \xi_{n, k}=\frac{1}{2^{\nu-1} \Gamma(\nu+1)} \frac{q_{n, k}^{\nu-1}}{J_{\nu+1}\left(q_{n, k}\right)}, \text { and } \\
& q_{n, k} \text { are the positive zeros of } J_{\nu}(\cdot) .
\end{aligned}
$$

As $t \rightarrow \infty$, the hazard function satisfies:

$$
\mathcal{T}(0) \cdot \lim _{t \rightarrow \infty} h(t)=\frac{q_{n, 1}^{2}}{2 n}>\max \left\{1, \frac{(n-1)^{2}}{2 n}\right\} .
$$

Proposition 5 compares the asymptote of the hazard rate with the expected time until adjustment, which equals $\mathcal{T}(0)=\bar{y} /\left(n \sigma^{2}\right)$, as derived above. Notice that for a model with a constant hazard, these two quantities are the reciprocal 


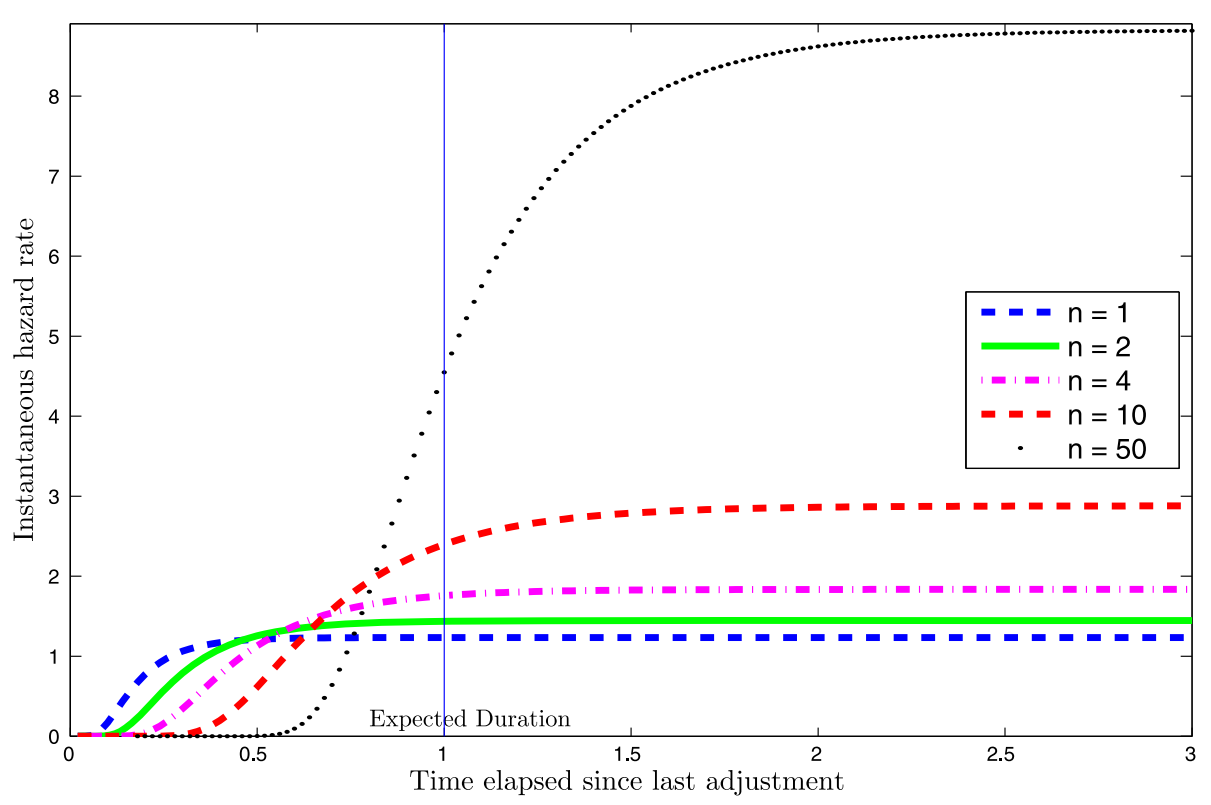

FIGURE 1.- Hazard rate of price adjustments for various choices of $n$. For each $n$, the value of $\sigma^{2} / \bar{y}$ is chosen so that the expected time elapsed between adjustments is 1 .

of each other, that is, the expected duration is the reciprocal of the hazard rate. We use the product $\mathcal{T}(0) \cdot \lim _{t \rightarrow \infty} h(t)$, which is greater than 1 , as a measure of how close the model is to having a constant hazard rate. Also notice that the expression in Proposition 5 immediately shows that, keeping the expected time until adjustment $\mathcal{T}(0)$ fixed, the hazard rate is only a function of $n$. Thus, the shape of the hazard function depends only on the number of products $n$. Changes in $\sigma^{2}, B$, and $\psi$ only stretch the horizontal axis linearly.

Figure 1 plots the hazard rate function $h$ for different choices of $n$ keeping the expected time between price adjustment fixed at 1 . As Proposition 5 shows, the function $h$ has an asymptote, which is increasing in the number of products $n$. Moreover, since the asymptote diverges to $\infty$ as $n$ increases with no bound, the hazard rate converges to an inverted L shape, as the hazard for a model where adjustment occurs exactly every $\mathcal{T}(0)=1$ periods as in Taylor's (1980) model. To see this, note that defining $\tilde{y} \equiv y / \bar{y}$ and fixing the ratio $\mathcal{T}(0)=\bar{y} /\left(n \sigma^{2}\right)$ so that for any $n$, the expected time elapsed between price changes is $\mathcal{T}(0)$, we have

$$
\mathrm{d} \tilde{y}=\frac{1}{\mathcal{T}(0)} \mathrm{d} t+2 \sqrt{\tilde{y} \frac{1}{n \mathcal{T}(0)}} \mathrm{d} \mathcal{W} \quad \text { for } \quad \tilde{y} \in[0,1]
$$


As $n \rightarrow \infty$, the process for the normalized size of the price gap $\tilde{y}$ described in equation (12) converges to a deterministic process, in which case the hazard rate is zero between times 0 and below $\mathcal{T}(0)$, and is $\infty$ precisely at $\mathcal{T}(0)$.

The shape of estimated hazard rates varies across studies, but many investigators have found flat or decreasing hazards; some have found hump-shaped ones. As can be seen from Figure 1, the hazard rate for the case of $n=1$ is increasing but rapidly reaches its asymptote. As $n$ increases, the shape of the hazard rate becomes closer to the inverted L shape of its limit as $n \rightarrow \infty$. For instance, when $n=10$, the level of the hazard rate evaluated at the expected duration is about twice the hazard for $n=2$. This is a prediction that can be tested in the cross section using the data set in Bhattarai and Schoenle (2011) or Wulfsberg (2010).

Next we characterize the marginal distribution of price changes. The reason to focus on the marginal distribution is that it corresponds to what is measured in the data, where no record is kept of the joint distribution of price changes. This distribution is characterized by two parameters: the number of goods $n$ and the optimal boundary of the inaction set $\bar{y}$. The value of $\bar{y}$, as discussed above, depends on all the parameters. Let $\tau$ be a time when $y$ hits the boundary of the range of inaction: since after an adjustment, all price gaps are reset to zero, the price changes coincide with $\Delta p(\tau)=-p(\tau)$, where $p(\tau) \in \partial \mathcal{I} \subset \mathbb{R}^{n}$, that is, the price vector belongs to the surface of an $n$-dimensional sphere of radius $\sqrt{\bar{y}}$. Given that each of the (uncontrolled) $p_{i}(t)$ is independently and identically normally distributed, the price changes $\Delta p(\tau)=-p(\tau)$ are uniformly distributed on the $n$-dimensional surface of the sphere. ${ }^{9}$ We can now state the following result.

PROPOSITION 6: Let $p \in \partial \mathcal{I} \subset \mathbb{R}^{n}$ denote a vector of price gaps on the boundary of the inaction region, triggering price changes $\Delta p=-p$. The distribution of the price change of an individual good $i$, that is, the marginal distribution of $\Delta p_{i} \in[-\sqrt{\bar{y}}, \sqrt{\bar{y}}]$, has density

$$
w\left(\Delta p_{i}\right)=\frac{1}{\operatorname{Beta}\left(\frac{n-1}{2}, \frac{1}{2}\right) \sqrt{\bar{y}}}\left(1-\left(\frac{\Delta p_{i}}{\sqrt{\bar{y}}}\right)^{2}\right)^{(n-3) / 2},
$$

where $\operatorname{Beta}(\cdot, \cdot)$ denotes the Beta function. The standard deviation and kurtosis of the price changes, the expected value of the absolute value of price changes, and its coefficient of variation are given by

$$
\operatorname{Std}\left(\Delta p_{i}\right)=\sqrt{\bar{y} / n}, \quad \operatorname{Kurt}\left(\Delta p_{i}\right) \equiv \frac{\mathbb{E}\left(\Delta p_{i}\right)^{4}}{\left(\mathbb{E}\left(\Delta p_{i}\right)^{2}\right)^{2}}=\frac{3 n}{n+2},
$$

\footnotetext{
${ }^{9} \mathrm{To}$ see this, notice that the probability density function (p.d.f.) of a jointly normally distributed vector of $n$ identical and independent normals is given by a constant times the exponential of the square radius of the sphere, divided by half of the common variance.
} 


$$
\begin{aligned}
& \mathbb{E}\left[\left|\Delta p_{i}\right|\right]=\frac{\sqrt{\bar{y}}}{\frac{n-1}{2} \operatorname{Beta}\left(\frac{n-1}{2}, \frac{1}{2}\right)}, \\
& \frac{\operatorname{Std}\left(\left|\Delta p_{i}\right|\right)}{\mathbb{E}\left(\left|\Delta p_{i}\right|\right)}=\sqrt{\left[\frac{n-1}{2} \operatorname{Beta}\left(\frac{n-1}{2}, \frac{1}{2}\right)\right]^{2} \frac{1}{n}-1 .}
\end{aligned}
$$

As $n \rightarrow \infty$, the distribution of $\Delta p_{i} / \operatorname{Std}\left(\Delta p_{i}\right)$ converges pointwise to a standard normal.

The proof uses results from the characterization of spherical distributions by Song and Gupta (1997); see Appendix A. Using Proposition 6 and the approximation for $\bar{y}$, we obtain the expression $\operatorname{Std}\left(\Delta p_{i}\right)=\left(\frac{\sigma^{2} \psi}{B} \frac{2(n+2)}{n^{2}}\right)^{1 / 4}$, which shows that the standard deviation of price changes is decreasing in $n$, while the kurtosis of the price changes is increasing in $n .^{10}$

Proposition 6 establishes how the shape of the distribution of price changes $w\left(\Delta p_{i}\right)$ varies substantially with $n$, as shown in Figure 2. For $n=2$, the distribution is $\mathrm{U}$-shaped (with density diverging toward infinity at the boundaries); for $n=3$, it is uniform; for $n=4$, it has the shape of a half circle; and for $n \geq 6$, it has a bell shape. Proposition 6 also establishes that as $n \rightarrow \infty$, the distribution converges to a normal. This can be seen in Figure 2 by comparing the distribution for $n=50$ with a normal distribution with the same standard deviation and a zero mean. Interestingly, the expressions in Proposition 6 show that $w\left(\Delta p_{i}\right)$ and $\left|\Delta p_{i}\right|$ depend only on $n$ and on the scale of the distribution,
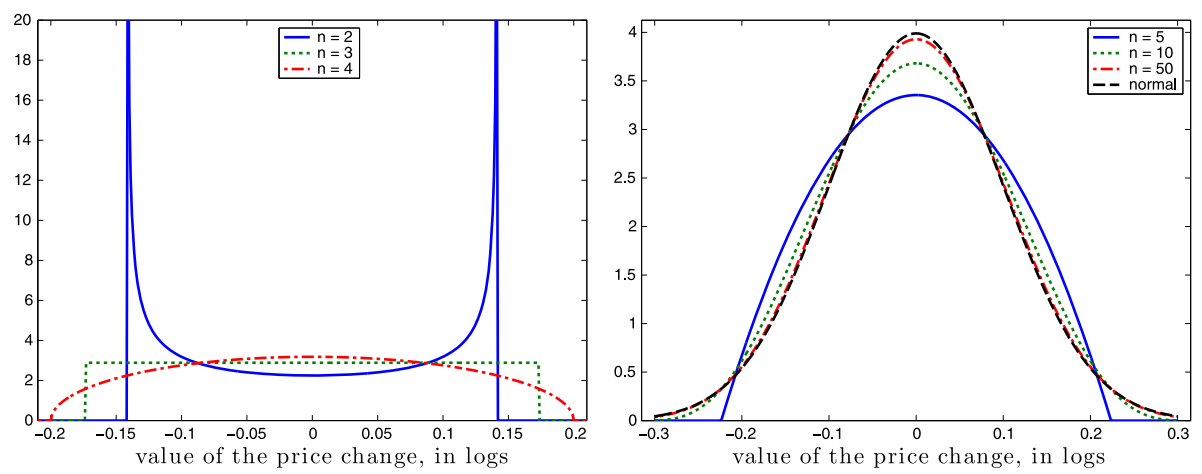

FIGURE 2.-Density of the price changes for various choices of $n: w\left(\Delta p_{i}\right)$. All distributions have the same standard deviation of price changes: $\operatorname{Std}\left(\Delta p_{i}\right)=0.10$.

\footnotetext{
${ }^{10}$ The approximations $\mathbb{E}\left[\left|\Delta p_{i}\right|\right] \approx \operatorname{Std}\left(\Delta p_{i}\right) \sqrt{\frac{2}{\pi}\left(1+\frac{1}{2 n}\right)}$ and $\frac{\operatorname{Std}\left(\left|\Delta p_{i}\right|\right)}{\mathbb{E}\left(\left|\Delta p_{i}\right|\right)} \approx \sqrt{\frac{\pi}{2}\left(\frac{2 n}{1+2 n}\right)-1}$ are useful
} to see how these statistics vary with $n$. 
TABLE I

Statistics for Price Changes as a Function of Number of Products: MODEL ECONOMY ${ }^{\mathrm{a}}$

\begin{tabular}{llllllllll}
\hline \hline & \multicolumn{10}{c}{ Number of Products $n$} \\
\cline { 2 - 11 } Statistics & \multicolumn{1}{c}{1} & \multicolumn{1}{c}{2} & \multicolumn{1}{c}{3} & 4 & 5 & 6 & 10 & 20 & 50 \\
\hline $\operatorname{Std}\left(\left|\Delta p_{i}\right|\right) / \mathbb{E}\left(\left|\Delta p_{i}\right|\right)$ & 0 & 0.48 & 0.58 & 0.62 & 0.65 & 0.67 & 0.70 & 0.74 & 0.75 \\
Kurtosis $\left(\Delta p_{i}\right)$ & 1.0 & 1.5 & 1.8 & 2.0 & 2.1 & 2.3 & 2.5 & 2.8 & 2.9 \\
Fraction: $\left|\Delta p_{i}\right|<\frac{1}{2} \mathbb{E}\left(\left|\Delta p_{i}\right|\right)$ & 0 & 0.21 & 0.25 & 0.27 & 0.28 & 0.28 & 0.30 & 0.31 & 0.31 \\
Fraction: $\left|\Delta p_{i}\right|<\frac{1}{4} \mathbb{E}\left(\left|\Delta p_{i}\right|\right)$ & 0 & 0.10 & 0.12 & 0.13 & 0.14 & 0.14 & 0.15 & 0.16 & 0.16 \\
\hline
\end{tabular}

\footnotetext{
${ }^{\mathrm{a}} \Delta p_{i}$ denotes the log of the price change and $\left|\Delta p_{i}\right|$ denotes the absolute value of the log of price changes. All
} statistics in the table depend exclusively on $n$.

$\sqrt{\bar{y}}$. Thus, any normalized statistic, such as ratio of moments (kurtosis, skewness, etc.) or a ratio of points in the cumulative distribution function (c.d.f.), depends exclusively on $n$. This property can be used to parametrize or estimate the model.

We conclude this section by summarizing the most interesting results of the model concerning the cross-section predictions in comparison with the data and with the previous literature. To this end, Table I uses the model to compute several moments of interest that depend only on $n$. Table II reports the empirical counterparts to those moments as estimated by Midrigan (2011) (using two scanner data sets) and by Bhattarai and Schoenle (2011) (using Bureau of Labor Statistics (BLS) producer data).

First, in comparison with the menu cost models of Golosov and Lucas (2007) or Midrigan (2011), the model's ability to account for the shape of the distribution of price changes improves dramatically. These models predict a bimodal distribution of price changes with a nil, or very small, mass of small

TABLE II

Statistics for Price Changes as a Function of the Number of Products: U.S. Data a

\begin{tabular}{|c|c|c|c|c|c|c|c|c|}
\hline \multirow[b]{3}{*}{ Statistics } & \multirow{2}{*}{\multicolumn{4}{|c|}{$\frac{\text { Bhattarai and Schoenle }}{\text { Number of Products } n}$}} & \multicolumn{4}{|c|}{ Midrigan } \\
\hline & & & & & \multicolumn{2}{|c|}{ AC Nielsen } & \multicolumn{2}{|c|}{ Dominick's } \\
\hline & 2 & 4 & 6 & 10 & All & No Sales & All & No Sales \\
\hline $\operatorname{Std}\left(\left|\Delta p_{i}\right|\right) / \mathbb{E}\left(\left|\Delta p_{i}\right|\right)$ & 1.02 & 1.15 & 1.30 & 1.55 & 0.68 & 0.72 & 0.84 & 0.81 \\
\hline $\operatorname{Kurtosis}\left(\Delta p_{i}\right)$ & 5.5 & 7.0 & 11 & 17 & 3.0 & 3.6 & 4.1 & 4.5 \\
\hline Fraction: $\left|\Delta p_{i}\right|<\frac{1}{2} \mathbb{E}\left(\left|\Delta p_{i}\right|\right)$ & 0.39 & 0.45 & 0.47 & 0.50 & 0.24 & 0.25 & 0.34 & 0.31 \\
\hline Fraction: $\left|\Delta p_{i}\right|<\frac{1}{4} \mathbb{E}\left(\left|\Delta p_{i}\right|\right)$ & 0.27 & 0.32 & 0.35 & 0.38 & 0.10 & 0.10 & 0.17 & 0.14 \\
\hline
\end{tabular}

\footnotetext{
${ }^{\text {a }}$ For the Bhattarai and Schoenle (2011) data, the number of products $n$ is the mean of the categories considered based on the information in Table 1, the ratio $\operatorname{Std}\left(\left|\Delta p_{i}\right|\right) / \mathbb{E}\left(\left|\Delta p_{i}\right|\right)$ is from Table 2 (firm-based), the fraction of $\left|\Delta p_{i}\right|$ that are small is from Table 14, and the kurtosis is from Figure 7. The data from Midrigan (2009) are taken from the distribution of standardized prices in Table $2 b$.
} 
price changes, as can be seen by the $n=1$ and the $n=2$ cases in Figure 2 . In contrast, as documented by Klenow and Malin (2010) as well as Midrigan (2011), the size distribution of price changes has a bell shape and displays a large mass of small price changes, as shown in Table II. We showed that the number of goods that is necessary to produce the bell-shaped distribution is at least 6 . Therefore, compared to the U.S. data in Table II, the models with $n=1,2$ generate too few small price changes. Much larger values of $n$ are necessary to reproduce the patterns that are seen in the data.

Another novelty of the model is that, in addition to producing a larger mass of small price changes compared to existing models, it also produces a greater mass of "large" price changes, so that its kurtosis is higher than is obtained in models with small $n$. This is seen immediately by noting that the kurtosis is $3 n /(2+n)$, an increasing function of $n$. The multiproduct hypothesis is thus able to account for more kurtosis than the canonical menu cost model. We notice, however, that despite this improvement, the model is not yet able to match the very high level of kurtosis that is measured by some data sets. We see this as a challenge, both theoretical and empirical, for future work. ${ }^{11}$

The model provides a simple explanation for the empirical regularity documented by Goldberg and Hellerstein (2009) and Bhattarai and Schoenle (2011) using BLS producer prices that firms selling more goods (or larger firms) tend to adjust prices more frequently and by smaller amounts. This is precisely what our model predicts based on equation (11) and Proposition 6. Notice that the prediction holds even if, in doing the comparative statics with respect to $n$, one assumes that the fixed cost $\psi$ increases linearly with $n .{ }^{12}$

Finally, there is quite a bit of evidence that, as in our model, price changes tend to be synchronized across products sold by the same firm, as in the seminal paper by Lach and Tsiddon (1996), who showed that price changes are synchronized within stores but staggered across stores. In our model, synchronization is perfect, that is, all the products sold by the firm change prices simultaneously, while in the data, the synchronization is less than perfect. Additionally, it is found empirically that similar products tend to be more synchronized; see Levy, Bergen, Dutta, and Venable (1997) and Dutta, Bergen, Levy, and Venable (1999), and Cavallo (2010), who calls them "synchronization in the aisle." This suggests that we can interpret the $n$ goods in our model as a subset of similar products sold by a firm (an "aisle"). A discussion of this evidence, as well as additional results by Fisher and Konieczny (2000), Chakrabarti and Scholnick (2007), Midrigan (2009, 2011), Bhattarai and Schoenle (2011), and Anderson,

\footnotetext{
${ }^{11}$ The level of kurtosis appears to be imprecisely measured in the data: the estimates vary widely from around 3 to 20 , depending on the data source, industry, sample selection criteria, and measurement error as discussed by Eichenbaum, Jaimovich, Rebelo, and Smith (2012) and Alvarez, Le Bihan, and Lippi (2013).

${ }^{12}$ See the National Bureau of Economic Research (NBER) version of this paper (Alvarez and Lippi (2012)) for a discussion of this point and some empirical evidence.
} 
Jaimovich, and Simester (2012), is given in the Appendix D in the Supplemental Material.

\section{THE RESPONSE TO A MONETARY SHOCK}

In this section, we study the response of the aggregate price level to an unexpected permanent monetary shock. Understanding this impulse response is useful to quantify the real effects of monetary shocks in the presence of menu costs, to identify its determinants, and to characterize how the effects vary with the number of products $n$ sold by the firm. We will show how the determinants of the real effects of monetary shocks map into simple observable statistics about the size and frequency of price changes that are available for many economies.

A main novelty of this paper is to solve the whole impulse response analytically using an approximation to characterize the firm's decision problem. In particular, in addition to the second order approximation of the profit function and the assumption of no drift in the price gaps used above, we assume that after an aggregate monetary shock, the firm uses the same decision rule $\bar{y}$ used in the steady state, that is, we ignore the feedback effect on the firm's decision that arises in a general equilibrium problem. Interestingly, we show that the approximation provides a very accurate benchmark to the exact solution of the original problem. The explanation for this result, formally stated in Proposition 7 and numerically evaluated in the Appendix C in the Supplemental Material, is that in the class of models we considered, the general equilibrium feedback only has second order effects on the decision rules.

The general equilibrium setup where we embed our price-setting problem is an adaptation of the Golosov and Lucas (2007) model to multiproduct firms (see Appendix B in the Supplemental Material for details). The representative household has preferences given by

$$
\begin{aligned}
& \int_{0}^{\infty} e^{-r t}\left(u(c(t))-\alpha \ell(t)+\log \frac{M(t)}{P(t)}\right) d t, \quad \text { and } \\
& c(t)=\left(\int_{0}^{1} \sum_{i=1}^{n}\left(Z_{k, i}(t) c_{k, i}(t)\right)^{(\eta-1) / \eta} d k\right)^{\eta /(\eta-1)},
\end{aligned}
$$

where $u(c)=\left(c^{1-\varepsilon}-1\right) /(1-\varepsilon), c_{k, i}(t)$ is the consumption of product $i$ produced by firm $k, \ell(t)$ is labor services, $M(t)$ is the nominal quantity of money, $P(t)$ is the nominal ideal price index of one unit of aggregate consumption, and $r>0, \varepsilon \geq 1, \alpha>0$, and $\eta>1$ are parameters. The elasticity of substitution between any two products $\eta$ is the same, regardless of the firms that produced them..$^{13}$ The production function for good $i$ in firm $k$ at time $t$ is linear in labor (the only input in the economy) with productivity $1 / Z_{k i}(t)$, so the

\footnotetext{
${ }^{13}$ See Section 6 for the case where the elasticities are different.
} 
marginal cost of that product is $W(t) Z_{k i}(t)$, where $W(t)$ is the nominal wage. We assume that the idiosyncratic productivity and demand shocks are perfectly correlated, and that $Z_{k i}(t)=\exp \left(\sigma \mathcal{W}_{k i}(t)\right)$, where $\mathcal{W}_{k i}$ is standard BM independent across all $k, i$. This assumption ensures that consumer expenditure is the same across goods so that the ideal price index is well defined and has equal weights. Firm $k$ can adjust one or more of its $n$ nominal prices by paying a fixed menu cost equal to a number of labor service units, which we express as $\psi$ times the steady state profits from producing one good evaluated at the profit maximizing price. Markets are complete and all firms are owned by the representative household. We use $R(t), W(t)$, and $P_{k i}(t)$ for the time $t$ nominal interest rate, nominal wage, and nominal price of firm $k$ on product $i$, respectively. As before, we use $p_{k i}(t)$ for the price gap, that is, the log of the ratio of the nominal price of firm $k$ on product $i$ to the frictionless optimal price:

$$
p_{k i}(t)=\log P_{k i}(t)-\log \left(W(t) Z_{k i}(t)\right)-\log (\eta /(\eta-1)) .
$$

We consider an economy that starts at the invariant distribution of firm's prices that correspond to a steady state with constant money supply equal to $\bar{M}$. We assume that at time $t=0$, there is an unanticipated permanent increase in the level of the money supply by $\delta \log$ points, so $\log M(0)=\log \bar{M}+\delta$, where bars denote the steady state values. As in the general equilibrium sticky price model of Danziger (1999) or Golosov and Lucas (2007), we have that for all $t \geq 0$, the interest rate is constant and wages and consumption follow ${ }^{14}$

$$
R(t)=r, \quad \log \frac{W(t)}{\bar{W}}=\delta, \quad \log \frac{c(t)}{\bar{c}}=\frac{1}{\varepsilon}\left(\delta-\log \frac{P(t)}{\bar{P}}\right) .
$$

Equation (15) shows that the shock induces an immediate permanent increase in (the log of) nominal wages, and hence marginal cost, by $\delta$. The effect on output, on the other hand, is gradual and at each $t$, it depends on how much the aggregate price level $P(t)$ is.

The next proposition illustrates why the approximate decision rule $\bar{y}$, which was derived in a partial equilibrium from the quadratic loss function $\mathbf{V}(\boldsymbol{\tau}, \boldsymbol{\Delta} \mathbf{p} ; p)$ in equation (1), provides an accurate approximation of the effect of a monetary shock $\delta$ in a general equilibrium. To this end, recall that $\{\boldsymbol{\tau}, \boldsymbol{\Delta} \mathbf{p}\} \equiv\left\{\boldsymbol{\tau}_{j}, \Delta p_{i}\left(\tau_{j}\right)\right\}_{j=1}^{\infty}$ denotes the stochastic processes for the stopping times and the $n$ price gaps, and let $\mathbf{c} \equiv\{c(t) / \bar{c}-1\}_{t \geq 0}$ denote the path of aggregate output deviations from the steady state. Let the value function $-\mathcal{V}(\boldsymbol{\tau}, \boldsymbol{\Delta} \mathbf{p}, \mathbf{c} ; p)$ measure the expected profits in the general equilibrium of a generic firm with a vector of price gaps $p$.

\footnotetext{
${ }^{14}$ The first two equations follow from the assumptions of log utility for real balances and linear utility of leisure, as in Golosov and Lucas (2007). The nominal and real rates are equal since money growth is zero.
} 
Proposition 7: Let $\delta \geq 0$. For all $t \geq 0$, a general equilibrium satisfies the following statements:

(i)

$$
\begin{aligned}
\log \frac{P(t)}{\bar{P}}= & \delta+\int_{0}^{1}\left(\sum_{i=1}^{n}\left(p_{k i}(t)-\bar{p}_{k i}\right)\right) d k \\
& +\int_{0}^{1}\left(\sum_{i=1}^{n} o\left(\left\|p_{k i}(t)-\bar{p}_{k i}\right\|\right)\right) d k
\end{aligned}
$$

(ii) $A$ Taylor expansion of $\mathcal{V}(\boldsymbol{\tau}, \boldsymbol{\Delta} \mathbf{p}, \mathbf{c} ; p)$ around $p_{i, t}=0$ for all $i=1, \ldots, n$ and around $c_{t}=\bar{c}$ is proportional to the quadratic loss function $\mathbf{V}(\boldsymbol{\tau}, \boldsymbol{\Delta} \mathbf{p} ; p)$. Moreover, the terms including the cross-products of the price gap $p_{i t}$ and the aggregate consumption $c_{t}$ are of third order or higher.

Part (i) states that the effect of the shock on $P(t)$ can be approximated by analyzing the dynamic response of the price gaps, each of which falls by a constant $\delta$ before any adjustment takes place, that is, $p_{k i}(0)=\bar{p}_{k i}-\delta$. Part (ii) states that the objective function $\mathbf{V}$ in the partial equilibrium setup of equation (1) is proportional to the objective function in the general equilibrium setup $\mathcal{V}$. The difference between these functions in terms of the price gaps (the relevant object for the firm's decision) only involves third and higher order terms, so that the general equilibrium effect on the boundary of the inaction set, $\bar{y}$, is second order. The result provides a foundation to Caballero and Engel (1991, 1993, 2007), who pioneered the analytical study of the impulse response in $S s$ models while ignoring the general equilibrium feedback effects on the decision rules. To see why the result holds, note that, as Golosov and Lucas (2007) remark, the general equilibrium feedback on the firms' decision rules is completely captured at each time $t$ by the effect of the aggregate output $c(t)$ on profits. Inspection of the firm's profit function shows that the discounted period $t$ profits from good $i$ are the product of a term that includes the general equilibrium effect - a function of $c(t)$ - and a term whose maximum is achieved by the frictionless price. ${ }^{15}$ Hence the discounted time $t$ profits have a first derivative with respect to the price gap $p_{i}(t)$ equal to zero, and have a second cross-derivative with respect to the price gap $p_{i}(t)$ and $c(t)$, which is also zero. The zero crossderivative of the profit function implies that the firm's price-setting decisions do not depend on the aggregate variables (up to first order), beyond the effect of the monetary shock on wages. Moreover, the equilibrium behavior of the nominal wages implies that the law of motion of the price gaps following a

\footnotetext{
${ }^{15}$ This profit function follows by two assumptions of the Golosov-Lucas setup discussed above: first, preferences for real balances and leisure imply that nominal wages are a function of money stock; second, the constant elasticity of substitution (CES) demand for the firm's products and the constant returns to scale technology imply the multiplicative structure of profits.
} 
shock is the same as the behavior of the steady state. The consequence of these assumptions is that for small shocks $\delta$ and small adjustment $\operatorname{cost} \psi$-so that the order of approximation using the above argument is valid-the firm's pricing decisions following a monetary shock differ from the steady state decision only up to second order terms, that is, the general equilibrium effect on the firms' pricing decisions is negligible (see Appendix B in the Supplemental Material for a proof and Appendix C in the Supplemental Material for a quantitative exploration of this result).

Next we use the results of Proposition 7 to study the effect of an aggregate monetary shock of size $\delta$ on the aggregate price level $P(t)$ at $t \geq 0$ periods after the shock, which we denote by $\mathcal{P}_{n}(\delta, t)$. As commonly done in the sticky price literature, we characterize the first order approximation to the price index, so, in particular, we study $\mathcal{P}_{n}(\delta, t) \equiv \delta+\int_{0}^{1}\left(\sum_{i=1}^{n} p_{k i}(t)\right) d k \approx \log P(t) / \bar{P}$. Once we characterize the effect on the price level, we describe the effect on employment and output. The impulse response is made of two parts: an instantaneous impact adjustment (a jump) of the aggregate price level that occurs at the time of the shock, denoted by $\Theta_{n}(\delta)$, and a continuous flow of adjustments from $t>0$ on, denoted by $\theta_{n}(\delta, t)$. The cumulative effect of the price level $t \geq 0$ periods after the shock is

$$
\mathcal{P}_{n}(\delta, t)=\Theta_{n}(\delta)+\int_{0}^{t} \theta_{n}(\delta, s) d s .
$$

We also study the impact effect on the fraction of firms that change prices, denoted by $\Phi_{n}(\delta)$.

We focus on the cumulative price response because its difference with the monetary shock, $\delta-\mathcal{P}_{n}(\delta, t)$, is proportional to the aggregate output at time $t$, as discussed in Section 5.5. Next we present our main results on $\mathcal{P}_{n}(\delta, t)$ and $\Phi_{n}(\delta)$ following an aggregate shock.

PROPOSITION 8: Fix n, the number of goods sold by each firm.

(i) Parameters. The impulse response $\mathcal{P}_{n}(\delta, t)$ depends only on two parameters, $\sqrt{\bar{y}}$ and $\sigma$, which we reparameterize as functions of two steady state statistics: the standard deviation of price changes $\operatorname{Std}\left[\Delta p_{i}\right]$ and the frequency of price changes $N_{a}$.

(ii) Scaling and Stretching. The IRF of an economy with steady state $\operatorname{Std}\left[\Delta p_{i}\right], N_{a}$ and a shock $\delta$ at horizon $t \geq 0$ is a scaled version of the response of an economy with unit steady state parameters, normalized monetary shock $\delta / \operatorname{Std}\left[\Delta p_{i}\right]$, and $a$ stretched horizon $N_{a} t$ :

$$
\mathcal{P}_{n}\left(\delta, t ; N_{a}, \operatorname{Std}\left[\Delta p_{i}\right]\right)=\operatorname{Std}\left[\Delta p_{i}\right] \mathcal{P}_{n}\left(\frac{\delta}{\operatorname{Std}\left[\Delta p_{i}\right]}, N_{a} t ; 1,1\right) .
$$

(iii) Impact Effects. The impact effects $\mathcal{P}(\delta, 0)=\Theta_{n}(\delta)$ and $\Phi_{n}(\delta)$ are strictly increasing in $\delta$, they are, respectively, strictly below $\delta$ and 1 , in the interval 
$\left(0,2 \operatorname{Std}\left[\Delta p_{i}\right]\right)$ and achieve these values outside this interval. Moreover, the impact effects are second order in the monetary shock: $\Theta_{n}^{\prime}(0)=\Phi_{n}^{\prime}(0)=0$.

Part (i) of Proposition 8 provides a reparameterization of the impulse response that is interesting for three reasons: (a) the steady state statistics $\operatorname{Std}\left[\Delta p_{i}\right]$ and $N_{a}$ are readily available for actual economies, (b) the results of Section 4 imply that, even fixing $n$, one can always choose two parameters values of $\psi / B$ and $\sigma^{2}$ to match these two statistics, and (c) by keeping fixed these two observable statistics and just changing $n$, we can isolate completely the role of the number of products $n$.

Part (ii) of Proposition 8 states a useful "scaling" property of the impulse response function. First, notice that at $t=0$, the impact effect of a monetary shock $\Theta_{n}$ is the same for any two economies with the same steady state average size of price changes $\operatorname{Std}\left[\Delta p_{i}\right]$ and is independent of the value of the steady state frequency of price adjustment $N_{a}$. Moreover, for all times following the impact $(t>0)$, the effect of a monetary shock $\delta$ in an economy characterized by steady state statistics $\operatorname{Std}\left[\Delta p_{i}\right]$ and $N_{a}$ depends only on $n$. This means that for a fixed $n$, the whole profiles of the impulse response functions in economies with different values of $\operatorname{Std}\left[\Delta p_{i}\right]$ and $N_{a}$ are simply scaled version of each other. For instance, fixing $n, \delta$, and $\operatorname{Std}\left[\Delta p_{i}\right]$, the impulse response functions in two economies that differ in the frequency of price adjustments, say $N_{a}$ vs. $2 N_{a}$, will have exactly the same values of $\mathcal{P}_{n}$, but will reach these values at different times, respectively, $2 t$ vs. $t$, that is, an economy with twice as many flexible prices in steady state has an impulse response that reaches each value in half the time. Furthermore, keeping $N_{a}$ fixed, the height of the whole impulse response function $\mathcal{P}_{n}$ is proportional to the scaled value of the monetary shock. This property of the impulse response function is also convenient to aggregate across the sectors of an economy with different frequencies of adjustment and sizes of price changes. We find this characterization interesting in itself, that is, even interesting for the $n=1$ case, but more importantly it will allow us to compare the impulse responses for economies that feature different values of $n$.

Part (iii) of Proposition 8 shows that the size of the monetary shock matters. For large shocks, there is instantaneous full price flexibility $\left(\Theta_{n}=\delta\right)$, but for small shocks, the size of the initial jump in price is second order compared to the shock. This, together with part (ii), implies that whether a monetary shock is large or not is completely characterized by comparing it with the typical price change in steady state, that is, it is a function of $\delta / \operatorname{Std}\left[\Delta p_{i}\right]$.

For the reader who is not interested in the derivation of the impulse responses and an explanation of the different effects behind it, we include two figures that summarize the quantitative conclusions of our analysis. Before getting to these figures, we note that its computation for large values of $n$ would have been extremely costly without the characterization given in the sections below. Figure 3 has two panels that illustrate the impact effect on 

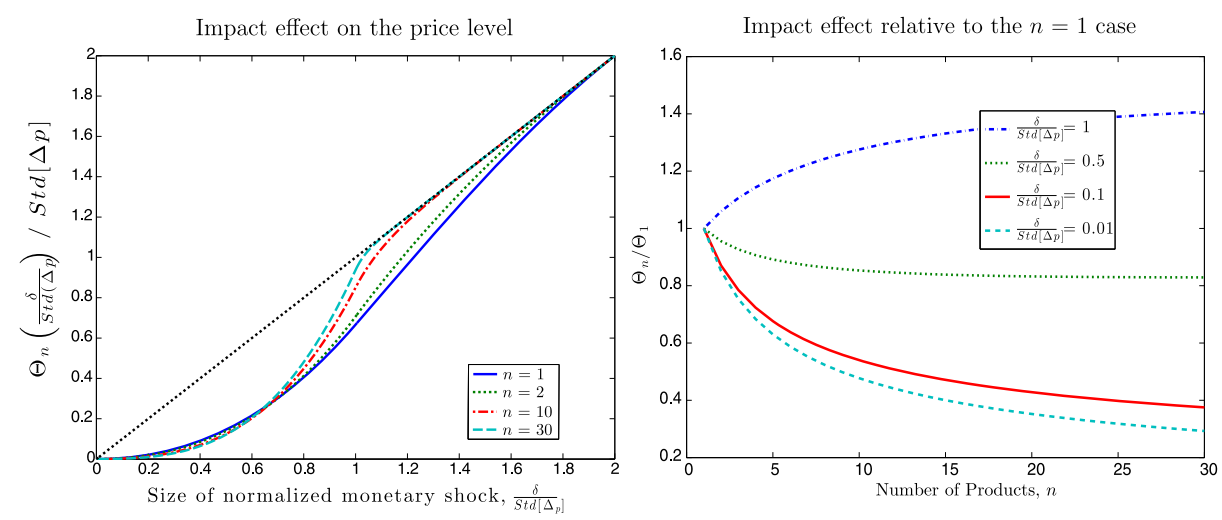

FIGURE 3.-The impact effect of an aggregate shock on the price level: $\Theta_{n}$.

prices of monetary shocks of different sizes for economies with different values of $n$. The left panel shows the normalized impact on the aggregate price level, $\Theta_{n} / \operatorname{Std}\left[\Delta p_{i}\right]$ (on the vertical axis), of a normalized monetary shock, that is, a shock $\delta / \operatorname{Std}\left[\Delta p_{i}\right]$ (on the horizontal axis). Each line plotted in this panel corresponds to a different number of products $n$. Recall that if $\Theta_{n}(\delta)=\delta$, the shock is neutral, and that, instead, when $\Theta_{n}(\delta)<\delta$, the shock implies an increase in real output. As stated in Proposition 8 , if $\delta \geq 2 \sqrt{\bar{y} / n}=2 \operatorname{Std}\left[\Delta p_{i}\right]$, then all firms adjust prices and hence the shock is neutral. This explains the range of the normalized shock between 0 and 2. For the quantification of this figure, it is helpful to notice that on the one hand, a typical estimate of the standard deviation of price changes for U.S. or European countries is $10 \%$ or higher, that is, $\operatorname{Std}\left[\Delta p_{i}\right] \approx 0.1$. On the other hand, to quantify $\delta$, note that in a short interval-say a month—changes of the money supply or prices on the order of $1 \%$ are very rare. ${ }^{16}$ This figure also shows that for small $\delta$, the aggregate price effects are of order $\delta^{2}$, as stated in Proposition 8. Interestingly, the impact response of a monetary shock changes order with respect to $n$ as the value of $\delta$ increases, as can be seen for shocks smaller than a threshold $\delta / \operatorname{Std}\left[\Delta p_{i}\right] \approx 0.7$. Note that using $\operatorname{Std}\left[\Delta p_{i}\right]=0.1$ implies that the order is reversed for monetary shocks $\delta$ higher than 7\%, a very large value. The right panel of Figure 3 displays four lines, each corresponding to a different value of $\delta$. Each line shows the aggregate effect on prices as $n$ changes, relative to the $n=1$ case. From these two panels, it can be seen that for monetary shocks on the order of those experienced by economies with inflation close to zero, that is, for increases in money $\delta / \operatorname{Std}\left[\Delta p_{i}\right]$ smaller than 0.5 (or for the benchmark value, for $\delta$ smaller than $5 \%$ ), economies with more products are stickier than those with fewer.

\footnotetext{
${ }^{16}$ For instance, the monthly innovations on a time series representation of M1 are on the order of 50 basis points, and that is without any conditioning. Presumably exogenous monetary shocks are much smaller.
} 


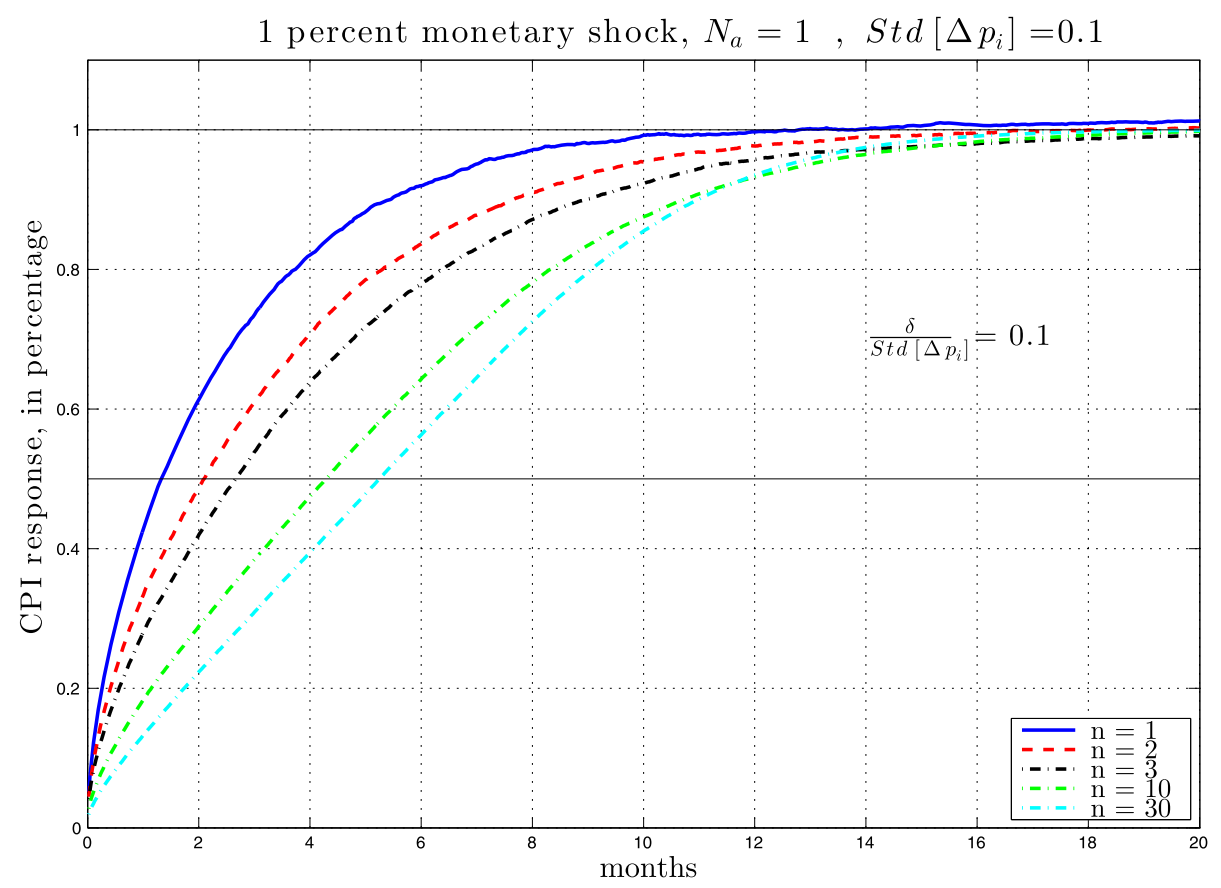

FIGURE 4.--Impulse response of the aggregate price level.

Figure 4 plots the impulse response function $\mathcal{P}_{n}(\delta, t)$ for economies with different $n$, keeping fixed the steady state deviation of price changes to $10 \%$, that is, $\operatorname{Std}\left[\Delta p_{i}\right]=0.1$, and an average of one price change per year, that is, $N_{a}=1$. The size of the monetary shock is $1 \%$, so that $\delta / \operatorname{Std}\left[\Delta p_{i}\right]=0.1$. In Figure 4, we time aggregated the effect on the aggregate price level up to daily periods. As required, all impulse responses display an impact effect on the first period and a monotone convergence to the full adjustment of the shock. The impact effect of the monetary shock during the first period is to increase prices about $5 \%$ of the long run value (i.e., five basis points) for $n=1$. This effect is smaller in economies where firms produce more products, that is, the impact at $t=0$ is decreasing in $n$. This difference is small between one and two products, but the effect is almost halved for firms with 10 products, as shown in Figure 3 for a monetary shock of the same size.

Likewise, the shape and duration of the shocks depend on $n$. The half-life of the shock more than doubles as the number of products goes from 1 to 10 . The shape of the impulse responses for $n=1$ is quite concave, but for large $n$ it becomes almost linear, up to a value of $t$ of about $1 / N_{a}$. This pattern of the shape is consistent with the result of Proposition 5, which shows that for large $n$ the model becomes a version of either the Taylor (1980) staggering price model or the Caballero (1989), Bonomo and Carvalho (2004), and Reis (2006) inatten- 
tiveness model, where the staggering lasts for $\mathcal{T}(0)=1 / N_{a}$ periods. Indeed, in Proposition 12 below, we show that as $n \rightarrow \infty$, the impulse response becomes linear up to time $1 / N_{a}$ because there is no "selection effect." Summarizing, we find that extending the analysis from $n=1$ to a larger number of product (say $n \approx 10$ ) almost halves the impact effect on the aggregate price level and doubles the half-life of the shock for empirically reasonable monetary shocks. ${ }^{17}$

The rest of this section is organized as follows. First, we obtain a closed form solution for the IRF in the case of $n=1$. This result, which is novel and interesting in its own right, is also helpful to better understand the derivation for the $n \geq 2$ case as well as to compare the results. After that, we develop the analytical expressions for the $n \geq 2$ case, concentrating first on the impact effects and then on the remaining part of the impulse response. The proof of Proposition 8, as well as explicit expressions for the impulse responses, are presented as separate propositions in the next subsections. We conclude the section by discussing the real effect of the monetary shocks.

\subsection{Impulse Response for the $n=1$ Case}

In the $n=1$ case, which we refer to (slightly abusing the analogy) as the Golosov and Lucas (GL) case, the firm controls the price gap between two symmetric thresholds, $\pm \bar{p}$, and when the price gap hits either threshold, resets the price gap $p$ to zero. Hence the invariant distribution of price gaps is triangular: the density function has a maximum at $p=0$ and decreases linearly on both sides to reach a value of zero at the thresholds $\bar{p}$ and $-\bar{p}$, since firms that reach the thresholds will adjust upon a further shock. An example of such a distribution is depicted by the solid line in the left panel of Figure 5. A straight-
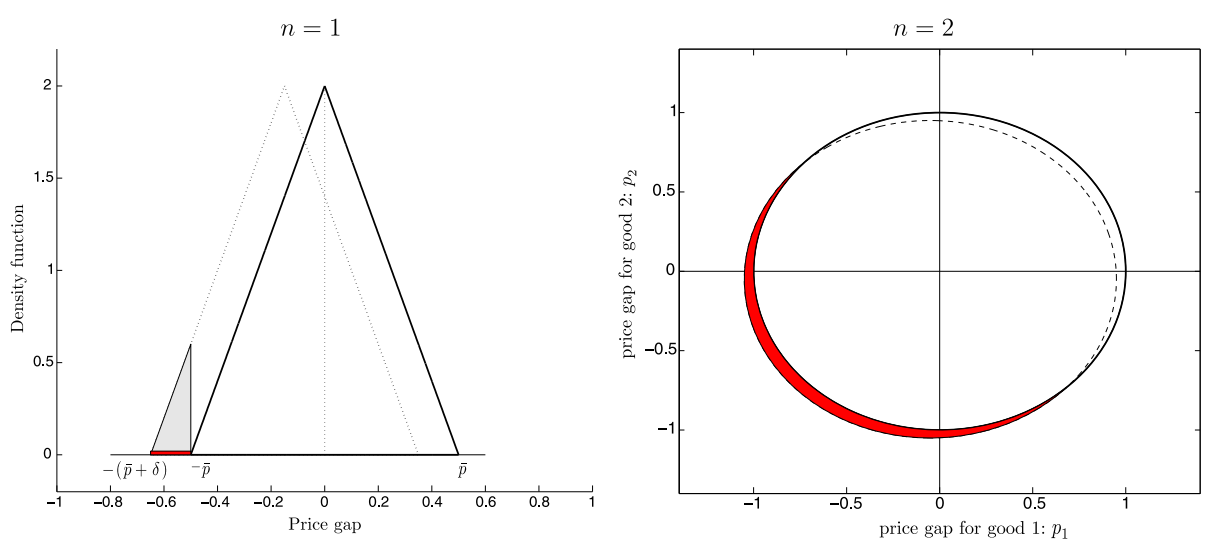

FIGURE 5.-The selection effect on impact for the $n=1$ and $n=2$ cases.

\footnotetext{
${ }^{17}$ The results are very similar for shocks of $1 / 2$ and 2\%; see Appendix F3 in Alvarez and Lippi (2012).
} 
forward computation gives that the slope of this density is $\pm(1 / \bar{p})^{2}$. Consider an aggregate shock that displaces the distribution by reducing all price gaps by $\delta$. If the value of $\delta>2 \bar{p}$, then all the firms will adjust their price, so that $\Phi=1$, and after a simple calculation, one can see that the aggregate price level is increased by $\delta$. Instead, if the value of $\delta$ is smaller than $2 \bar{p}$, only the firms with a sufficiently small price gap will adjust. Denoting the price gap right after the shock by $p_{0}$, these are the firms that end up with $p_{0}<-\bar{p}$. The density of the distribution of the price gaps immediately after the shock, denoted by $\lambda$, is depicted by the dotted line of Figure 5 and is given by

$$
\lambda\left(p_{0}, \delta ; \bar{p}\right)= \begin{cases}\frac{1}{\bar{p}}\left(1+\frac{\delta}{\bar{p}}+\frac{p_{0}}{\bar{p}}\right) & \text { if } \frac{p_{0}}{\bar{p}} \in\left[-1-\frac{\delta}{\bar{p}},-\frac{\delta}{\bar{p}}\right], \\ \frac{1}{\bar{p}}\left(1-\frac{\delta}{\bar{p}}-\frac{p_{0}}{\bar{p}}\right) & \text { if } \frac{p_{0}}{\bar{p}} \in\left(-\frac{\delta}{\bar{p}}, 1-\frac{\delta}{\bar{p}}\right] .\end{cases}
$$

For a shock of size $\delta$, the mass of such firms is $\Phi=(1 / 2)(\delta / \bar{p})^{2}$, which uses the slope of the density given above (to simplify notation, we suppress the $n=$ 1 subindex). Note that the magnitude of this fraction is proportional to the square of the shock, a feature that is due to the fact that there are a few firms close to the boundary of the inaction set. This case is depicted by the dotted line in the left panel of Figure 5. Firms that change prices "close the price gap" completely, so that the price increase will be $\delta+\bar{p}$ for the firm that prior to the shock had price gap $-\bar{p}$ and it will be equal to $\bar{p}$ for the firm with preshock price gap equal to $-\bar{p}+\delta$. Using the triangular distribution of price gaps, we have that the average price increase among those firms that adjust prices equals $\bar{p}+\delta / 3$. Let us denote by $\Theta$ the impact effect on aggregate prices of a monetary shock of size $\delta$, the product of the number of firms that adjust times the average adjustment among them. Note that in steady state, the average size of price changes, as measured by the standard deviation of price changes $\operatorname{Std}\left[\Delta p_{i}\right]$, is given by $\bar{p}$. Thus for $\delta \leq \operatorname{Std}\left[\Delta p_{i}\right]=\bar{p}$, we can write

$$
\Theta=\operatorname{Std}\left[\Delta p_{i}\right] \frac{1}{2}\left(\frac{\delta}{\operatorname{Std}\left[\Delta p_{i}\right]}\right)^{2}\left(1+\frac{1}{3} \frac{\delta}{\operatorname{Std}\left[\Delta p_{i}\right]}\right),
$$

so that for an economy with one good, the impact effect on prices, normalized by the steady state average price change, depends on the normalized monetary shock and is locally quadratic, at least for a small shock. Note that the degree of aggregate stickiness is independent of the steady state fraction of price changes.

We now develop an expression for the impulse response at horizons $t>0$. The density of the price gaps $p_{0}$ right after the monetary shock $\delta$ is the displaced triangular distribution $\lambda$ plotted in Figure 5 and described in equation (17), and hence it has $\bar{p}$ as a parameter. It peaks at $-\delta$ and has support $[-\bar{p}-\delta, \bar{p}-\delta]$. Note that the impact adjustment is concentrated on the 
firms whose price gap is smaller than $-\bar{p}$. Now consider the contribution to the change in aggregate prices of the firms whose price gap is $p_{0} \in[-\bar{p}, \bar{p}-\delta]$, so they have not adjusted on impact, and of which there are $\lambda\left(p_{0} ; \delta\right) d p_{0}$. Let $G^{-}\left(t ; p_{0}\right)$ be the probability that a firm with price gap $p_{0}$ at time zero will increase price before time $t$, that is, the probability that its price gap will hit $-\bar{p}$ before time $t$ without first hitting $\bar{p}$. Likewise define $G^{+}\left(t ; p_{0}\right)$ as the corresponding probability of a price decrease, let $G\left(t ; p_{0}\right)=G^{-}\left(t ; p_{0}\right)-G^{+}\left(t ; p_{0}\right)$ be the difference between these probabilities, and let $g$ be its density. We note that these functions have $\left(\bar{p}, \sigma^{2}\right)$ as parameters. We can now define the contribution to the change in the price level of the adjustments that take place between $t$ and $t+d t$ as ${ }^{18}$

$$
\theta(\delta, t)=\bar{p} \int_{-\bar{p}}^{\bar{p}-\delta} g\left(t ; p_{0}\right) \lambda\left(p_{0} ; \delta\right) d p_{0} .
$$

The integral excludes the initial price gaps $p_{0}$ that are below $-\bar{p}$. These correspond to firms that adjusted on impact. Note that $\theta(\delta, t)$ have $\left(\bar{p}, \sigma^{2}\right)$ as parameters. Expressions for the densities $g^{+}$and $g^{-}$can be found in equations (15) and (16) of Kolkiewicz (2002). This gives

$$
\begin{aligned}
g\left(t ; p_{0}\right)= & \frac{\sigma^{2}}{2 \bar{p}^{2}} \sum_{k=1}^{\infty} e^{-\left(k^{2} \pi^{2} /\left(2 \bar{p}^{2}\right)\right) \sigma^{2} t} k \pi \\
& \times\left[\sin \left(k \pi\left(1+\frac{p_{0}}{\bar{p}}\right)\right)-\sin \left(k \pi\left(1-\frac{p_{0}}{\bar{p}}\right)\right)\right] .
\end{aligned}
$$

Four remarks are in order. First, by substituting our expressions for $g$ and $\lambda$, we have a closed form solution for each expression in equation (18). Second, note that we did not need to compute the evolution of the whole cross-sectional distribution. Instead, we follow each firm until the first time that it adjusted its price. This is because the subsequent adjustments have a zero net contribution to aggregate prices, since after the adjustment, every firm's price gap returns to zero and the subsequent adjustments are as likely to be increases as decreases. Third, note that the role of the monetary shock is just to displace the initial distribution, that is, $\delta$ is not an argument of $g$. Fourth, note that this function has two interesting forms of homogeneity. The first type of homogeneity is that it is homogeneous of degree 1 in $\sigma, \bar{p}$, and $\delta$. This follows because scaling $\bar{p}$ and $\delta$ will just scale proportionally the distribution $\lambda$ of the initial price gaps.

\footnotetext{
${ }^{18}$ Alternatively one could compute the impulse response for the $n=1$ case by adapting ideas from Bertola and Caballero (1994). They studied the evolution of the whole cross-section distribution following a shock for an irreversible investment problem with a reflecting barrier. Their formulas should be adapted to our fixed cost problem whose optimal return point implies a jump (not a reflection) of the state.
} 
Furthermore, scaling $\bar{p}$ and $\sigma$ keeps the probabilities of hitting any two scaledup values in the same elapsed time the same. The second type of homogeneity uses that a standard Brownian motion at time $t$ started at time zero has a normal distribution with variance $t$. So scaling the variance of the shock, the price gaps will hit any given value in a scaled time. These two homogeneity properties can be seen by integrating the previous expression to give an IRF that satisfies the properties stated in Proposition 8:

$$
\begin{aligned}
\mathcal{P}(\delta, t)= & \Theta(\delta)+\operatorname{Std}\left[\Delta p_{i}\right] \sum_{k=1}^{\infty} \frac{1-e^{-\left(k^{2} \pi^{2} / 2\right) N_{a} t}}{k \pi} \\
& \times \int_{-1}^{1-\delta / \operatorname{Std}(\Delta p)}[\sin (k \pi(1+x))-\sin (k \pi(1-x))] \\
& \times \lambda\left(x, \frac{\delta}{\operatorname{Std}\left[\Delta p_{i}\right]} ; 1\right) d x .
\end{aligned}
$$

\subsection{Invariant Distribution of $y=\|p\|^{2}$}

Here we study the invariant distribution of the sum of the squares of the price gaps $y \equiv\|p\|^{2}=\sum_{i=1}^{n} p_{i}^{2}(t)$ under the optimal policy. This will be used to describe the starting point of the economy before the monetary shock. We denote the density of the invariant distribution by $f(y)$ for $y \in[0, \bar{y}]$. This is of interest for studying the response of firms that are in the steady state to an unexpected shock to their target that displaces the price gaps uniformly. The density of the invariant distribution for $y$ is found by solving the corresponding forward Kolmogorov equation and the relevant boundary conditions (see Appendix A for the proof).

PROPOSITION 9: The density $f(\cdot)$ of the invariant distribution of the sum of the squares of the price gaps $y$, for given thresholds $\bar{y}$ in the case of $n \geq 1$ products, is, for all $y \in[0, \bar{y}]$,

$$
f(y)= \begin{cases}\frac{1}{\bar{y}}[\log (\bar{y})-\log (y)] & \text { if } n=2 \text { and } \\ (\bar{y})^{-n / 2}\left(\frac{n}{n-2}\right)\left[(\bar{y})^{n / 2-1}-(y)^{n / 2-1}\right] & \text { otherwise. }\end{cases}
$$

The density has a peak at $y=0$, decreases in $y$, and reaches zero at $\bar{y}$. The shape depends on $n$. The density is convex in $y$ for $n=1,2,3$, linear for $n=4$, and concave for $n \geq 5$. This is intuitive, since the drift of the process for $y$ increases linearly with $n$, hence the mass accumulates closer to the upper bound $\bar{y}$ as $n$ increases. Indeed, as $n \rightarrow \infty$, the distribution converges to a uniform in 
$[0, \bar{y}]$. Proposition 9 makes clear also that the shape of the invariant density depends exclusively on $n$; the value of the other parameters, $\psi / B, \sigma^{2}$ only enters in determining $\bar{y}$, which only stretches the horizontal axis proportionally.

\subsection{Impact Response in the $n \geq 2$ Case}

Now we turn to studying the economy-wide impact effect of the aggregate shock. To find out the fraction of firms that will adjust prices under the invariant, we need to characterize some features of the invariant distribution of $p \in \mathbb{R}^{n}$. We assume that the aggregate shock happens once and for all, so that the price gap process remains the same and the firms solve the problem stated above. First, we find out which firms choose to change prices and, averaging among their $n$ products, by how much. A firm with price gap $p \in \mathbb{R}^{n}$ and state $\|p\|^{2}=y \leq \bar{y}$ before the shock will have its price gaps displaced downward by $\delta$ in each of its $n$ goods, that is, its state immediately after the shock is $\left\|p-1_{n} \delta\right\|$, where $1_{n}$ is a vector of 1 's. This firm will change its prices if and only if the state will fall outside the range of inaction, that is, $\left\|p-1_{n} \delta\right\| \geq \bar{y}$, or, equivalently, if and only if

$$
\begin{aligned}
& \|p\|^{2}-2 \delta\left(\sum_{i=1}^{n} p_{i}\right)+n \delta^{2} \geq \bar{y} \quad \text { or } \\
& \frac{\sum_{i=1}^{n} p_{i}}{\sqrt{y}} \leq \nu(y, \delta) \equiv \frac{y-\bar{y}}{2 \delta \sqrt{y}}+n \frac{\delta}{2 \sqrt{y}} .
\end{aligned}
$$

Thus $\nu(y, \delta)$ gives the highest value for the sum of the $n$ price gaps for which a firm with state $y$ will adjust the price. The normalized sum of price gaps $\sum_{i=1}^{n} p_{i} / \sqrt{y}$ takes values on $[-\sqrt{n}, \sqrt{n}]$. The right panel of Figure 5 shows the $n=2$ case by plotting a circle centered at zero that contains all the preshock price gaps, and shows the "displaced" price gaps right after the $\delta$ shock, which are given by a circle centered at $(-\delta, \delta)$. The shaded area contains all the price gaps of the firms that, after the shock, will find it optimal to adjust their prices, that is, firms for which equation (20) holds.

A firm whose price gap $p$ satisfies equation (20), that is, a firm with $(1 / \sqrt{y}) \sum_{i=1}^{n} p_{i} \leq \nu(y, \delta)$, will change all of its prices. The mean price change, averaging across its $n$ products, is $\delta-(1 / n) \sum_{i=1}^{n} p_{i}{ }^{19}$ Thus we can determine the fraction of firms that change their prices and the amount by which they change them by analyzing the invariant distribution of the squared price gaps,

\footnotetext{
${ }^{19}$ Recall that $p_{i}$ are the price gaps. Thus, to set them to zero, the price changes must take the opposite sign. Moreover, since $\delta$ has the interpretation of a cost increase, it decreases the price gap and, hence, its correction requires a price increase.
} 
$f(y)$. Let $S(z)$ denote the cumulative distribution function of the sum of the coordinates of the vectors distributed uniformly in the $n$-dimensional unit sphere. Formally, we define $S: \mathbb{R} \rightarrow[0,1]$ as

$$
S(z)=\frac{1}{L\left(\mathbb{S}^{n}\right)} \int_{x \in \mathbb{R}^{n},\|x\|=1} \mathbf{I}\left\{x_{1}+x_{2}+\cdots+x_{n} \leq z\right\} L(d x),
$$

where $\mathbb{S}^{n}$ is the $n$-dimensional sphere and where $L$ denotes its $n-1$ Lebesgue measure. Note that $S(\cdot)$ is weakly increasing, that $0=S(-\sqrt{n}), S(0)=1 / 2$, $S(\sqrt{n})=1$, and that it is strictly increasing for $z \in(-\sqrt{n}, \sqrt{n})$. Remarkably, as shown in Proposition 10, the distribution of the sum of the coordinates of a uniform random variable in the unit $n$-dimensional sphere is the same, up to a scale, as the marginal distribution of any of the coordinates of a uniform random variable in the unit $n$-dimensional sphere (which we discussed in Proposition 6), that is, the c.d.f. satisfies

$$
\begin{aligned}
& S^{\prime}(z) \equiv s(z)=\frac{1}{\operatorname{Beta}\left(\frac{n-1}{2}, \frac{1}{2}\right) \sqrt{n}}\left(1-\left(\frac{z}{\sqrt{n}}\right)^{2}\right)^{(n-3) / 2} \\
& \quad \text { for } \quad z \in(-\sqrt{n}, \sqrt{n})
\end{aligned}
$$

for $n \geq 2$; for $n=1$, the c.d.f. $S$ has two points with mass $1 / 2$ at -1 and at +1 . Now we are ready to give expressions for the effect of an aggregate shock $\delta$. First, consider $\Phi_{n}$, the fraction of firms that adjust prices. There are $f(y) d y$ firms with state $y$ in the invariant distribution; among them, the fraction $S(\nu(y, \delta))$ adjusts. Integrating across all the values of $y$, we obtain the desired expression. Second, consider $\Theta_{n}$, the change in the price level across all firms. There are $f(y) d y$ firms with state $y$ in the invariant distribution; among them, we consider all the firms with normalized sum of price gaps less than $\nu(y, \delta)$, for which the fraction $s(z) d z$ adjust prices by $\delta-\sqrt{y} z / n$. Considering all the values of $y$, we obtain the relevant expression. This gives the following proposition.

PROPOSITION 10: Consider an aggregate shock of size $\delta$. The fraction of price changes on impact, $\Phi_{n}$, and the average price change across the $n$ goods among all the firms in the economy, $\Theta_{n}$, are given by

$$
\begin{aligned}
& \Phi_{n}(\delta)=\int_{0}^{\bar{y}} f(y) S(\nu(y, \delta)) d y, \\
& \Theta_{n}(\delta)=\delta \Phi_{n}(\delta)-\int_{0}^{\bar{y}} f(y) \frac{\sqrt{y}}{n}\left[\int_{-\sqrt{n}}^{\nu(y, \delta)} z s(z) d z\right] d y,
\end{aligned}
$$


where $s(\cdot)$ is given by equation (21), which depends on $n$, and where $f(\cdot)$ and $\nu(\cdot)$, which are also functions of $\bar{y}$ and $n$, are given in equation (19) and equation (20), respectively.

See Appendix A for the proof and see Appendixes F1 and F2 in Alvarez and Lippi (2012) for a closed form solution and a lemma with the analytical characterization of $\Theta_{n}$ and $\Phi_{n}$ stated in part (iii) of Proposition 8.

\subsection{Impulse Response at Horizons $t>0$ in the $n \geq 2$ Case}

We develop an expression for the impulse responses at horizon $t>0$ for the general case of $n \geq 1$, in particular, we derive an expression for the flow impact on the price level at horizon $t$, which we denote as $\theta_{n}(\delta, t)$. As in the case of one good, we start by describing the distribution of firms indexed by their price gaps, immediately after the monetary shock $\delta$ but before any adjustment takes place. The c.d.f. $\Lambda_{n}\left(p_{0}\right)$ gives the fraction of price gaps smaller than or equal to $p_{0}$ at time zero right after the impact adjustment caused by the monetary shock $\delta$. Note that $\Lambda_{n}\left(p_{0}\right) \leq \Theta_{n}(\delta)$ for all $p_{0}$. To understand this expression, let $\tilde{p}_{0} \in \mathbb{R}^{n}$ be the price gap before the monetary shock, which has $y=\left\|\tilde{p}_{0}\right\|^{2}$ distributed according to the density $f(y)$ described in equation (19). The price gaps with a given value $\left\|\tilde{p}_{0}\right\|^{2} \equiv y \leq \bar{y}$ have a uniform distribution on the sphere, so their density depends only on $\left\|\tilde{p}_{0}\right\|^{2}$. Right after the monetary shock, these price gaps become $p_{0}=\tilde{p}_{0}-\mathbf{1}_{n} \delta$, where $\mathbf{1}_{n}$ is an $n$-dimensional vector of 1's. So we have that the density of the distribution of the price gaps immediately after the monetary shock but before any adjustment is

$$
\lambda\left(p_{0}, \delta\right)=f\left(\left\|p_{0}+\mathbf{1}_{n} \delta\right\|^{2}\right) \frac{\Gamma(n / 2)}{\pi^{n / 2}\left\|p_{0}+\mathbf{1}_{n} \delta\right\|^{n-2}}
$$

and we recall that $f(y)=0$ for any $y>\bar{y}$. We note that $\lambda$ is a function of $\bar{y}$ and $\delta$, but it is independent of $\sigma^{2}$. The scaling factor reflects two features: the change in variables from $y$ to $\sqrt{y}$ and the surface area of this sphere is given by $2 \pi^{n / 2} y^{(n-1) / 2} / \Gamma(n / 2)$, which gives a measure of the vectors $p_{0}$ with squared norm equal to $y$.

The next step is to find the contribution of those firms with price gap $p_{0}$ to the change in aggregate prices at horizon $t$. As in the case of one good, it suffices to consider the contribution of those firms that have the first price change exactly at $t$. This is because all subsequent adjustments have a zero net contribution to prices, since after the adjustment, the firm starts with a zero price gap. Since firms adjust prices when $y$ reaches $\bar{y}$ at some time $t$, we use the distribution of the corresponding hitting times and place in the sphere. In particular, let $G\left(p ; t, p_{0}\right)$ denote the probability that a firm with price gap $p_{0}$ at time zero will hit the surface of a sphere of radius $\sqrt{\bar{y}}$ at time $t$ or before with a price gap smaller than or equal to $p$. Note that $G$ is a function of $\sigma^{2}$ and $\bar{y}$, but 
it is independent of $\delta$. Explicit expressions for the joint density $g$ of the hitting time $t$ and place $p$ can be found in Wendel (1980) and Yin and Wang (2009). When the price gap of the firm hits the sphere of radius $\sqrt{\bar{y}}$ with a price gap $p$, the average change of its $n$ prices is given by "closing" each of the $n$ price gaps, that is, the average price change is given by $-\left(p_{1}+\cdots+p_{n}\right) / n$. Thus $\theta$, the contribution to the change in aggregate prices at time $t$ after a shock $\delta$ at time zero as defined in equation (16), is given by

$$
\begin{aligned}
\theta_{n}(\delta, t)= & \int_{\left\|p_{0}\right\|^{2} \leq \bar{y}}\left[\int_{\|p\|^{2}=\bar{y}} \frac{-\left(p_{1}+p_{2}+\cdots+p_{n}\right)}{n} g\left(p, t, p_{0}\right) d p\right] \\
& \times \lambda\left(p_{0}, \delta\right) d p_{0} .
\end{aligned}
$$

Note that the outer integral is computed only for the firms that have not adjusted on impact, that is, for the price gaps $\left\|p_{0}\right\|^{2} \leq \bar{y}$. Given the knowledge of the closed form expressions for both $\lambda$ and $g$, we can compute the multidimensional integrals in $\theta_{n}(\delta, t)$ by Monte Carlo procedures.

We adapt the expression for the density $g$ of hitting times and places from Theorem 3.1 of Yin and Wang (2009) to the case of a BM with variance $\sigma^{2}$ to obtain the next proposition.

PROPOSITION 11: Fix $n \geq 2$. Then the impulse response can be written as

$$
\mathcal{P}_{n}(\delta, t)-\Theta_{n}(\delta)=\sum_{m=0}^{\infty} \sum_{k=1}^{\infty} e_{m, k}(\delta, \sqrt{\bar{y}}, n)\left[1-\exp \left(-\frac{q_{m, k}^{2}}{2 n} \frac{n \sigma^{2}}{\bar{y}} t\right)\right],
$$

where the coefficients $q_{m, k}$ are the ordered (positive) zeroes of the Bessel function $J_{m+n / 2-1}(\cdot)$. The coefficients $e_{m, k}(\cdot, \cdot, n)$ are homogeneous functions of degree 1 in $(\delta, \sqrt{\bar{y}})$ and do not depend on $\sigma^{2}$. Furthermore, $\sum_{m=0}^{\infty} \sum_{k=1}^{\infty} e_{m, k}(\delta, \cdot, \cdot)=\delta-$ $\Theta_{n}(\delta) \leq \delta$.

See Appendix A for the proof. Using the properties of $\Theta_{n}$ from Proposition 10 and the homogeneity property of $e_{m, k}$ in equation (26), one verifies part (i) and part (ii) of Proposition 8.

We end this section with a full characterization of the impulse response function in the limiting case in which $n \rightarrow \infty$. The next proposition shows that when $n$ is large, the impulse response is linear, as was mentioned above.

Proposition 12: Assume that $\delta<\operatorname{Std}\left(\Delta p_{i}\right)$. We let $n \rightarrow \infty$, adjusting $\bar{y}$ and $\sigma^{2}$ to keep $\operatorname{Std}\left(\Delta p_{i}\right)=\sqrt{\bar{y} / n}$ and $N_{a}$ fixed. Then the fraction of immediate adjusters $\Phi_{n}(\delta) \rightarrow\left(\delta / \operatorname{Std}\left(\Delta p_{i}\right)\right)^{2}$, the immediate impact in the price level $\Theta_{n}(\delta) \rightarrow \delta \Phi_{n}(\delta)$, and the impulse response becomes linear, that is, $\mathcal{P}_{n}(\delta, t) \rightarrow$ $\Theta_{n}(\delta)+\delta N_{a}$ t for $0<t<T \equiv\left(1-\Phi_{n}(\delta)\right) / N_{a}$ and $\mathcal{P}_{n}(\delta, t) \rightarrow \delta$ for $t \geq T$. Furthermore, the average price change across firms at every $0 \leq t \leq T$ is equal to $\delta$. 
See Appendix A for the proof. The impact effect on the price level is of the order $\delta^{3}$ and, hence, for small values of $\delta$, it is negligible compared to the impact for the $n=1$ case, that is, $\Theta_{n} / \Theta_{1} \downarrow 0$ for $\delta \downarrow 0$, as shown in Figure 3. Moreover, Figure 4 shows that the half-life of the shock is $\left(1 / 2-\left(\delta / \operatorname{Std}\left(\Delta p_{i}\right)\right)^{2}\right) / N_{a}$, which converges to $1 /\left(2 N_{a}\right)$ for small shocks. In Figure 4 , which describes a $1 \%$ shock in the money supply, the half-life is three times greater than that produced by the $n=1$ case. A main consequence of the large $n$ is that there is no selection effect. This is to be compared with the case of $n=1$, where the selection effect is strongest and where, in the periods right after the shock (small $t$ ), all price adjustments are price increases. The reason for the lack of selection when $n$ is large is that for a firm selling many products, there are, upon adjustment, many cancellations since some prices will be increased and others decreased, so that the average price change across the firms' goods is simply $\delta$.

\subsection{On the Output Effect of Monetary Shocks}

This section discusses how the impulse response for prices is informative about the interpretation, time profile, and size of the output effect of a monetary shock. In the general equilibrium setups discussed at the beginning of this section, the deviation of output from the steady state output level is proportional to the change of the real balances, $\delta-\mathcal{P}_{n}(\delta, t)$, as shown in equation (15) and common to the models of Golosov and Lucas (2007), Caplin and Leahy (1997), and Danziger (1999). From now on we refer to $\delta-\mathcal{P}_{n}(\delta, t)$ as the impulse response of output, which is the expression predicted by our model in the case of log preferences $(\varepsilon=1) .{ }^{20}$

The half-life of the output response is identical to the half-life of the price level only in the case in which $\Theta_{n}=0$, that is, when there is no jump of the price level on impact, a condition that holds for infinitesimal shocks. When the price level jumps on impact $\left(\Theta_{n}>0\right)$, the half-life of the output response is longer than the half-life of the price level. The reason is that the jump shortens the time required for the price level to reach its half-life value (i.e., $\delta / 2)$, whereas the half-life target of the real output effect, given by $\left(\delta-\Theta_{n}(\delta)\right) / 2$, shifts and so its half-life is longer. To picture this effect in Figure 4, notice that different impact levels (corresponding to, e.g., different values of $n$ ) do not shift the half-life line (whose position is at $0.5 \%$ ), but will shift the half-life line of the real output effect (this line is not drawn in the figure; it is above 0.5 and shifts upward as $\Theta_{n}$ increases). The impact effect on output also depends on the size of the shock: on one hand, for very large shocks, there is full price flexibility and, hence, no effect on output regardless of $n$; on the other hand, for small monetary shocks, the impact effect on prices is of order smaller than $\delta$ and, hence, the impact effect on output is approximately $\delta$ for all values of $n$.

${ }^{20}$ If $\varepsilon \neq 1$, then the effect on output should be divided by $\varepsilon$, as shown in equation (15). 
As a summary statistic of the real effect of monetary shock, we use the area under the impulse response for output, that is,

$$
\mathcal{M}_{n}(\delta)=\int_{0}^{\infty}\left(\delta-\mathcal{P}_{n}(\delta, t)\right) d t
$$

which can be interpreted as the cumulative effect on output following the shock. This measure combines the size of the output deviations from the steady state with the duration of these deviations. Since $\mathcal{P}_{n}(\delta, t)$ depends only on the parameters $\operatorname{Std}\left(\Delta p_{i}\right)$ and $N_{a}$, so does $\mathcal{M}_{n}(\delta)$. Because of the homogeneity of $\mathcal{P}_{n}(\delta, t)$ discussed in part (ii) of Proposition 8 and the way time $\left(N_{a} t\right)$ enters $\mathcal{P}_{n}(\delta, t)$ shown in Proposition 11, we can thus write

$$
\mathcal{M}_{n}\left(\delta ; N_{a}, \operatorname{Std}\left(\Delta p_{i}\right)\right)=\frac{\operatorname{Std}\left(\Delta p_{i}\right)}{N_{a}} \mathcal{M}_{n}\left(\frac{\delta}{\operatorname{Std}\left(\Delta p_{i}\right)} ; 1,1\right),
$$

so that the effect of a shock of size $\delta$ in an economy characterized by parameters $\operatorname{Std}\left(\Delta p_{i}\right)$ and $N_{a}$ can be readily computed using the "normalized" effect for an economy with unit parameters and a standardized shock.

The determinants of the real effects of monetary shocks identified by equation (27) offer a new insight to measure the degree of aggregate price stickiness in menu cost models. The previous literature focused almost exclusively on the frequency of price changes, $N_{a}$, as a measure of stickiness, but equation (27) shows that the dispersion of price changes, $\operatorname{Std}\left(\Delta p_{i}\right)$, is also an important determinant. Indeed the area under the impulse response of output is proportional to the ratio of these two quantities, where the constant of proportionality depends on the (normalized) size of the monetary shock, $\delta / \operatorname{Std}\left(\Delta p_{i}\right)$ and, in our setup, on the number of products $n$.

Figure 6 illustrates how the real output effect of a monetary shock varies with the size of the shock $(\delta)$ and the number of goods sold by the firm $(n)$. The figure plots the summary impact measure as a function of $\delta$ for an economy with $\operatorname{Std}\left(\Delta p_{i}\right)=0.10$ and $N_{a}=1$, for four values of $n$. It is shown that for each value of $n$, the cumulative real effect of a monetary shock is hump-shaped in the size of the shock $(\delta)$. The effect is nil at extremes, that is, at $\delta=0$ and at $2 \operatorname{Std}\left(\Delta p_{i}\right)$ (not shown), as a reflection of the fact that large shocks induce full price flexibility (see part (iii) of Proposition 8 ). We characterize the value of $\delta$ for which $\mathcal{M}$ is maximized using equation (27) and the Figure 6. For a given $n$, the monetary shock that maximizes the cumulative output effect is about one-half of $\operatorname{Std}\left(\Delta p_{i}\right)$. Moreover, the maximum value of the cumulated output effect is proportional to $\operatorname{Std}\left(\Delta p_{i}\right) / N_{a}$. More interestingly, for the purpose of this paper, the size of the real effects varies with the number of goods $n$. Larger values of $n$, that is, firms selling more goods, produce larger cumulative effects for small values of the shock and also larger maximum values of the effect. In this sense, the stickiness of the economy is increasing in $n$. The maximum cumulative effect on output, on the order of $1.4 \%$ output points, is obtained as 


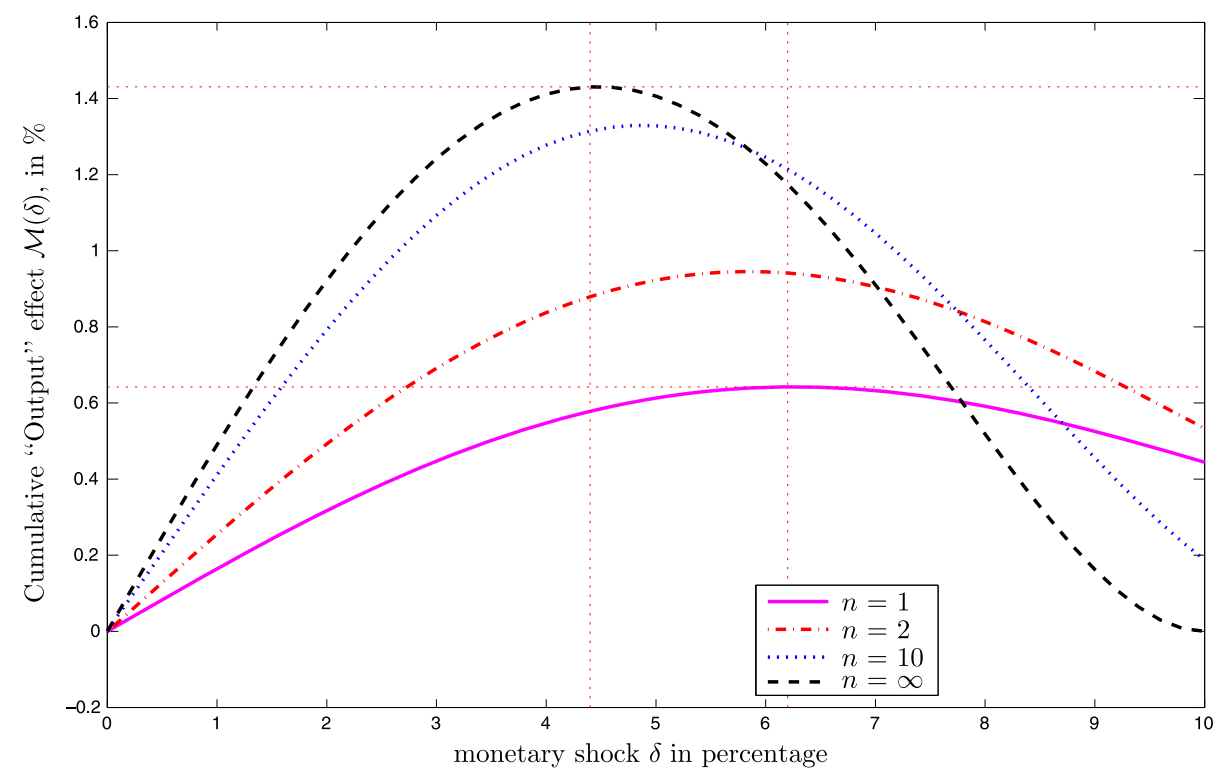

FigURE 6.-Cumulative output effect $\mathcal{M}(\delta)$. Parameters are $N_{a}=1$ and $\operatorname{Std}\left(\Delta p_{i}\right)=0.10$.

$n \rightarrow \infty$; a similar value obtains already for $n=10$. On the other hand, smaller effects are produced in models with $n=1$ or $n=2$.

\section{CORRELATION, INFLATION, AND DIFFERENT ELASTICITIES}

This section extends the baseline model to the case of price gaps $p_{i}, p_{j}$ that feature (i) cross-correlated innovations, (ii) a common drift (to model inflation), and (iii) cross-products $p_{i} p_{j}$ in the period return function of the firm (to model a demand system where the elasticity of substitution of products within the firm differs from the substitution elasticity of a product across firms). Surprisingly, despite the apparent complexity of these extensions, the modified problem remains tractable: instead of the scalar state variable $y$, the state of the problem with either drift, correlation, and/or cross-products includes only one additional variable to measure the sum of the coordinates of the vector, namely $z=\sum_{i=1}^{n} p_{i}$ for any $n$. For ease of exposition and because it turns out to be the one with more substantial effects, we focus here on the problem with correlated price gaps without drift and cross-products. We formulate the problem, derive its cross-section implications, and characterize the impulse response to a monetary shock. A fuller derivation of the results for correlation and its computations, as well as a derivation and analysis of the differential elasticity case, is given in Appendix E in the Supplemental Material.

The problem solved by the firm is, as before, the minimization of the value function in equation (1), subject to a law of motion for the $p_{i}$ that allows for 
correlation but no drift. The diffusions for the price gaps satisfy $\mathbb{E}\left[\mathrm{d} p_{i}^{2}(t)\right]=$ $\hat{\sigma}^{2} \mathrm{~d} t$ and $\mathbb{E}\left[\mathrm{d} p_{i}(t) \mathrm{d} p_{j}(t)\right]=\rho \hat{\sigma}^{2} \mathrm{~d} t$ for all $i=1, \ldots, n$ and $j \neq i$, and for two positive constants $\hat{\sigma}^{2}$ and $\rho$. Then we can write that each price gap follows $\mathrm{d} p_{i}(t)=\bar{\sigma} \mathrm{d} \overline{\mathcal{W}}(t)+\sigma \mathrm{d} \mathcal{W}_{i}(t)$ for all $i=1, \ldots, n$, where $\overline{\mathcal{W}}, \mathcal{W}_{i}(t)$ are independent standard BM's so that $\hat{\sigma}^{2}=\bar{\sigma}^{2}+\sigma^{2}$ and the correlation parameter is $\rho=\frac{\bar{\sigma}^{2}}{\bar{\sigma}^{2}+\sigma^{2}}$. Define $y(t)=\sum_{i=1}^{n} p_{i}^{2}(t)$ and $z(t)=\sum_{i=1}^{n} p_{i}(t)$, which by Ito's lemma obey the diffusions

$$
\begin{aligned}
\mathrm{d} y(t)= & n\left[\sigma^{2}+\bar{\sigma}^{2}\right] \mathrm{d} t+2 \sigma \sqrt{y(t)} \mathrm{d} \mathcal{W}^{a}(t)+2 \bar{\sigma} z(t) \mathrm{d} \mathcal{W}^{c}(t), \\
\mathrm{d} z(t)= & n \bar{\sigma} \mathrm{d} \mathcal{W}^{c}(t) \\
& +\sqrt{n} \sigma\left[\frac{z(t)}{\sqrt{n y(t)}} \mathrm{d} \mathcal{W}^{a}(t)+\sqrt{1-\left(\frac{z(t)}{\sqrt{n y(t)}}\right)^{2}} \mathrm{~d} \mathcal{W}^{b}(t)\right],
\end{aligned}
$$

where $\left(\mathcal{W}^{a}, \mathcal{W}^{b}, \mathcal{W}^{c}\right)$ are three standard (univariate) independent BM's. Notice that the introduction of correlation makes the variance of $y$ depend on the level of $z$.

In the case where $\sigma$ and $\bar{\sigma}$ are both positive, the state of this problem will be the pair $(y, z)$, and the value function, denoted by $v(y, z)$, is symmetric in $z$ around zero, so $v(y, z)=v(y,-z)$. The optimal policy is to have an inaction region $\mathcal{I}=\{(y, z): 0 \leq \bar{y}(z)\}$ for some function $\bar{y}(z)$ that satisfies $\bar{y}(z)=\bar{y}(-z)>0$ for all $z>0$. We solve $v(y, z)$ numerically for a problem with $r=0.05$ per year, $B=20$, and a volatility of each price gap of $13 \%$ with a pairwise correlation of $1 / 2$, so $\sigma=\bar{\sigma}=0.13 / \sqrt{2}$. The menu cost is $4 \%$ of frictionless profits per good, so $\psi / n=0.04$. We display the results for the case of $n=10$ products per firm.

The left panel of Figure 7 plots the value function over its $(y, z)$ domain. The value function region where control (i.e., price adjustment) is optimal is marked by bold dots. The feasible state space for the firm is the $y, z$ region inside the parabola in the right panel of the figure. For each $z$, the shape of the value function is similar to the case with no correlation. Fixing $y$, the value function is decreasing in $|z|$. This is because a higher $|z|$ implies a higher conditional variance of $y$ and thus a higher option value. Because of the higher option value, the threshold $\bar{y}(z)$ is increasing in $|z|$. While the inaction set is two dimensional, we emphasize that the state of the problem is $n$ dimensional: for instance, in the figure $n=10$.

We use the decision rule described above to produce the invariant distribution of a cross section of firms using simulations. The model parameterization is close to that used in the main body of the paper, that is, it produces a frequency of adjustments per year that is $N_{a}=1.3$ and a standard deviation $\operatorname{Std}\left(\Delta p_{i}\right)=0.11$. The left panel of Figure 8 plots the standardized distribution of price changes $w\left(\Delta p_{i}\right)$ for $n=2,3,50$ when the correlation between the shocks is $\rho=0.5$. The key effect of correlation is to increase the mass of price 

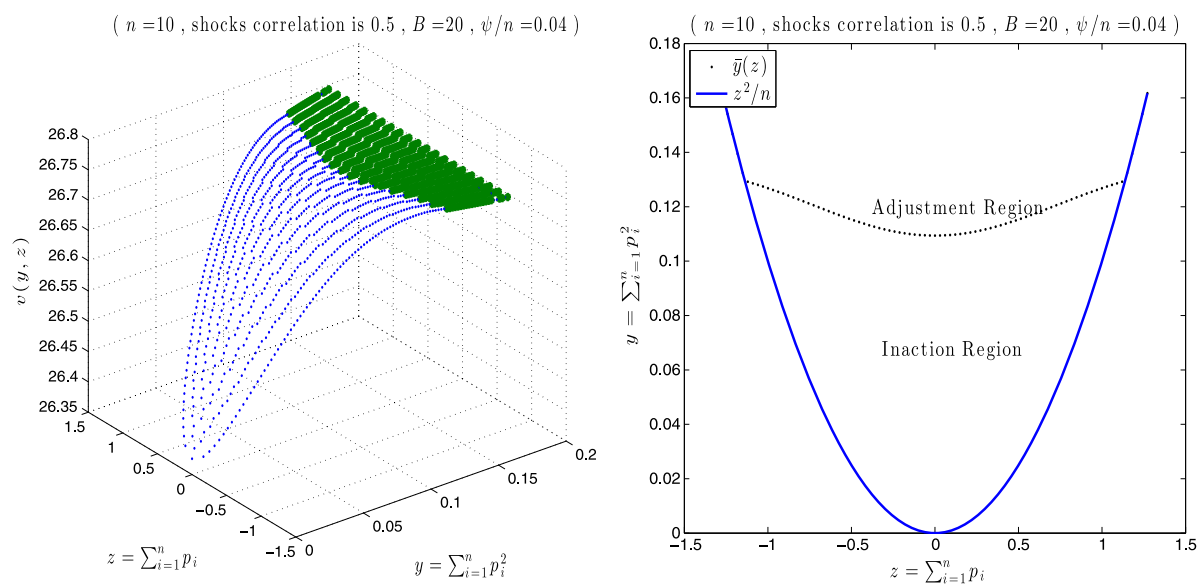

FIGURE 7.-Value function and decision rules with correlated shocks: $\rho=0.5$.

changes with similar sign, that is, to move mass from the center of the distribution toward both sides. Not surprisingly, adding correlation makes the model closer to the $n=1$ case, a feature that is important both for its empirical plausibility (i.e., the comparison with empirical distribution of price changes) and for the predicted effect of monetary shocks. The case of $n=3$ is particularly revealing, since for zero correlation, the distribution is uniform, but as the correlation is positive, the density becomes $U$-shaped, with a minimum at zero and two maxima at high values of the absolute value, as in the case of $n=1$. The case of $n=50$ is also informative because with zero correlation, this distribution is essentially normal. However, with positive correlation, the distribution
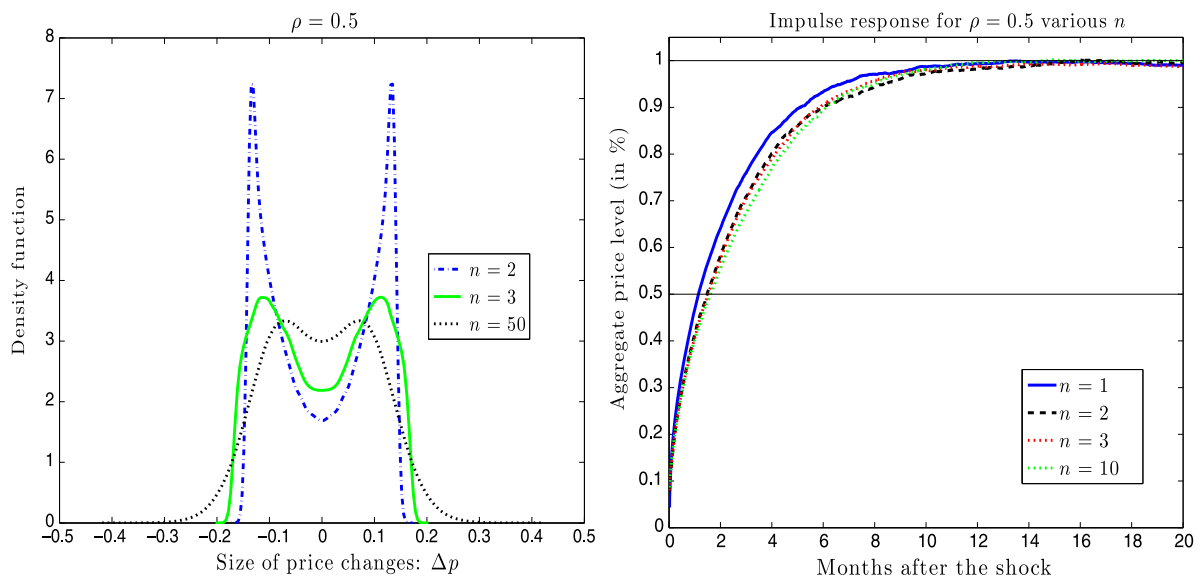

FIGURE 8.-The aggregate economy with correlated shocks: $\rho=0.5$. 
of price changes becomes bimodal, with a local minimum of its density at zero. Interestingly, the simultaneous near normality and bimodality (i.e., a small dip of the density around the center of the support) that is displayed by the $n=50$ case with correlation is apparent in several data sets, such as Midrigan (2009) (see his Figure 1, bottom two panels), Wulfsberg (2010) (see his Figure 4), and was explicitly tested and estimated by Cavallo and Rigobon (2010).

We conclude with the analysis of the price level response to a once and for all shock to the money supply in the presence of correlated shocks. We stress that to solve the IRF for any $n$, we only need to keep track of a two-dimensional object. This makes the procedure computationally feasible. We assume a correlation between shocks equal to $\rho=1 / 2$ for four economies with $n=1,2,3,10$. These economies are observationally equivalent in the steady state in terms of the price adjustment frequency $N_{a}$ and standard deviation $\operatorname{Std}\left(\Delta p_{i}\right){ }^{21}$ The impulse responses for $n=2,3,10$, displayed in Figure 8, show that introducing correlation significantly increases the price flexibility at all horizons: all impulse responses are now very close to the response produced by the model with $n=1$. This finding contrasts with the outcome that was obtained with no correlation in Figure 4. The intuition for this result is simple: introducing correlation increases the mass of "large" price changes, as was explained above. This effect brings back the "selection effect" that was being muted as $n$ got large in models with uncorrelated shocks.

\section{CONCLUDING REMARKS}

This paper presented a stylized model of price setting that substantially improves the cross-sectional predictions of menu cost models in comparison to the patterns that characterize the microdata. For instance, the model is able to produce a substantial mass of small price changes and a bell-shaped size distribution of price changes. The analytical tractability of the model allowed us to derive a full characterization of the steady state predictions as well as of the economy's aggregate response to a once and for all unexpected monetary shock, which we summarized in the Introduction.

We think that several extensions are interesting for future research. One feature of the data that our model misses concerns the kurtosis of price changes. In the model, the maximum level of kurtosis for the distribution of price changes predicted by the model is 3 , as in the Normal distribution. This value is larger than the prediction of the classical Barro (1972) or Dixit (1991) menu cost models (where kurtosis is 1), but it is still small compared to the large excess kurtosis detected in microdata sets. Larger values of the kurtosis can be

\footnotetext{
${ }^{21}$ Motivated by the scaling and stretching results of Proposition 8, we normalize the parameters so that the expected number of price changes per year is $1\left(N_{a}=1\right)$ and consider a shock of $10 \%$ of the size of the steady state standard deviation of price changes (namely $\delta=0.01$ and $\operatorname{Std}(\Delta p)=0.1$, that is, a $1 \%$ change in money supply and a $10 \%$ steady state standard deviation of price changes).
} 
obtained by introducing the possibility of random adjustment opportunities, as in models where the size of the menu cost is stochastic. We explored this problem in Alvarez, Le Bihan, and Lippi (2013) and showed that this assumption improves the empirical fit of the model cross section to the microdata and that it increases the real effect of a monetary shock by reducing the selection effect. Another interesting extension concerns the role of the linear production function (and no capital). The precision of our approximate solution benefited from this assumption since the firm's "optimal prices" did not depend on the level of the aggregate consumption. Instead, if production features decreasing returns to scale, the strength of the "pricing complementarities" increases, that is, the optimal price depends on the aggregate consumption. We leave it for the future to explore the quantitative importance of this alternative assumption.

\section{APPENDIX A: PROOFS}

ProOF OF PROPOSITION 2: The proof follows by substituting the function in equation (9) into the ODE (6) and matching the coefficients for the powers of $y^{i}$. By the Cauchy-Hadamard theorem, the power series converges absolutely for all $y>0$ since $\lim _{i \rightarrow \infty} \beta_{i+1} / \beta_{i}=0$. Notice that $v^{\prime}(0)=\beta_{1}$ and that $v(0)=$ $\beta_{0}$, so that we require $\beta_{1}>0$, which implies $\beta_{0}>0$. Moreover, if $\beta_{1}>B / r$, then $v$ is strictly increasing and strictly convex. If $\beta_{1}=B / r$, then $v$ is linear in $y$. If $0<\beta_{1}<B / r$, then $v$ is strictly increasing at the origin, strictly concave, and reaches its unique maximum at a finite value of $y$. Thus, a solution that satisfies smooth pasting requires that $0<\beta_{1}<B / r$ and the maximizer is $\bar{y}$. In this case, $y=0$ achieves the minimum in $[0, \bar{y}]$. Thus we have verified (i), (ii), and (iii).

Next we prove uniqueness. Let $\beta_{i}\left(\beta_{1}\right)$ be the solution of equation (9), as a function of $\beta_{1}$. Note that for $0<\beta_{1}<B / r$, all the $\beta_{i}\left(\beta_{1}\right)<0$ for $i \geq 2$ and are increasing in $\beta_{1}$, converging to zero as $\beta_{1}$ goes to $B / r$. Smooth pasting can be written as $0=v^{\prime}\left(\bar{y} ; \beta_{1}\right) \equiv \sum_{i=1}^{\infty} i \beta_{i}\left(\beta_{1}\right) \bar{y}^{i-1}$, where the notation emphasizes that all the $\beta_{i}$ can be written as a function of $\beta_{1}$. From the properties of the $\beta_{i}(\cdot)$ discussed above, it follows that we can write the unique solution of $0=v^{\prime}\left(\bar{\rho}\left(\beta_{1}\right) ; \beta_{1}\right)$ as a strictly increasing function of $\beta_{1}$, that is, $\bar{\rho}^{\prime}\left(\beta_{1}\right)>0$. The value matching condition at $\bar{y}$ gives $\psi=v\left(\bar{y}, \beta_{1}\right)-v\left(0, \beta_{1}\right)=$ $v\left(\bar{y}, \beta_{1}\right)-\beta_{0}\left(\beta_{1}\right)=\sum_{i=1}^{\infty} \beta_{i}\left(\beta_{1}\right) \bar{y}^{i}$. We note that, given the properties of $\beta_{i}(\cdot)$ discussed above, for any given $y>0$, we have that $v\left(y, \beta_{1}\right)-\beta_{0}\left(\beta_{1}\right)$ is strictly increasing in $\beta_{1}$ as long as $0<\beta_{1}<B / r$. Thus, define

$$
\Psi\left(\beta_{1}\right)=v\left(\bar{\rho}\left(\beta_{1}\right), \beta_{1}\right)-v\left(0, \beta_{1}\right)=\sum_{i=1}^{\infty} \beta_{i}\left(\beta_{1}\right) \bar{\rho}\left(\beta_{1}\right)^{i} .
$$

From the properties discussed above, we have that $\Psi\left(\beta_{1}\right)$ is strictly increasing in $\beta_{1}$ and that it ranges from 0 to $\infty$ as $\beta_{1}$ ranges from 0 to $B / r$. Thus $\Psi$ is invertible. The solution of the problem is given by setting $\beta_{1}(\psi)=$ $\Psi^{-1}(\psi)$ and $\bar{y}(\psi)=\bar{\rho}\left(\beta_{1}(\psi)\right)$.

Q.E.D. 
Proof of Proposition 3: Using the expression for $\left\{\beta_{i}\right\}$ obtained in Proposition 1 , the value matching and smooth pasting conditions can be written as two equations in $\beta_{2}$ and $\bar{y}$,

$$
\begin{aligned}
& \frac{\psi}{\bar{y}^{2}}=\frac{B}{r \bar{y}}+\beta_{2}\left[\frac{2 \sigma^{2}(n+2)}{r \bar{y}}+1+\sum_{i=1}^{\infty} \kappa_{i} r^{i} \bar{y}^{i}\right], \\
& 0=\frac{B}{r \bar{y}}+\beta_{2}\left[\frac{2 \sigma^{2}(n+2)}{r \bar{y}}+2+\sum_{i=1}^{\infty} \kappa_{i}(i+2) r^{i} \bar{y}^{i}\right],
\end{aligned}
$$

where $\kappa_{i}=r^{-i} \frac{\beta_{2+i}}{\beta_{2}}=\prod_{s=1}^{i} \frac{1}{\sigma^{2}(s+2)(n+2 s+2)}$. This gives an implicit equation for $\bar{y}$ :

$$
\psi=\frac{B}{r} \bar{y}\left[1-\frac{\frac{2 \sigma^{2}(n+2)}{r \bar{y}}+1+\sum_{i=1}^{\infty} \kappa_{i} r^{i} \bar{y}^{i}}{\frac{2 \sigma^{2}(n+2)}{r \bar{y}}+2+\sum_{i=1}^{\infty} \kappa_{i}(i+2) r^{i} \bar{y}^{i}}\right] .
$$

Since the right hand side is strictly increasing in $\bar{y}$ and goes from zero to infinity, then we obtain part (i). Since the right hand side is strictly decreasing in $n$ and goes to zero as $n \rightarrow \infty$, then we obtain part (ii). Rearranging this equation and defining $z=\bar{y} r / \sigma^{2}$ gives

$$
\begin{aligned}
& \frac{\psi 2(n+2)}{B \sigma^{2}} r^{2} \\
& =z^{2}+z^{3}\left[\frac{2(n+2) \sum_{i=1}^{\infty} \omega_{i}(i+1) z^{i-1}-2-\sum_{i=1}^{\infty} \omega_{i}(i+2) z^{i}}{2(n+2)+2 z+z \sum_{i=1}^{\infty} \omega_{i}(i+2) z^{i}}\right]
\end{aligned}
$$

where $\omega_{i}=\prod_{s=1}^{i} \frac{1}{(s+2)(n+2 s+2)}$. Using the expression for $\omega_{i}$ and collecting terms on $z^{i}$, one can show that the square bracket of equation (28) that multiplies $z^{3}$ is negative and, hence, $\bar{y}>\sqrt{\psi 2(n+2) \sigma^{2} / B}$. Letting $b=\psi r^{2} 2(n+2) /\left(B \sigma^{2}\right)$, we can write equation (28) as

$$
1=\frac{z^{2}}{b}\left(1+z\left[\frac{2(n+2) \sum_{i=1}^{\infty} \omega_{i}(i+1) z^{i-1}-2-\sum_{i=1}^{\infty} \omega_{i}(i+2) z^{i}}{2(n+2)+2 z+z \sum_{i=1}^{\infty} \omega_{i}(i+2) z^{i}}\right]\right) .
$$


Since $z \downarrow 0$ as $b \downarrow 0$, then $z^{2} / b \downarrow 1$ as $b \downarrow 0$, establishing part (iii). From equation (28), it is clear that the optimal threshold satisfies $\bar{y}=\frac{\sigma^{2}}{r} Q\left(\frac{\psi}{B \sigma^{2}} r^{2}, n\right)$. By differentiating this expression, we obtain part (iv).

Q.E.D.

ProOf OF PROPOSITION 5: The proof uses probability theory results on the first passage time of an $n$-dimensional Brownian motion (BM). Let $\tau$ be the stopping time defined by the first time when $\|p(\tau)\|^{2}$ reaches the critical value $\bar{y}$, starting at $\|p(0)\|=0$ at time zero. Let $S_{n}(t, \bar{y})$ be the probability distribution for times $t \geq \tau$. Alternatively, let $S_{n}(\cdot, \bar{y})$ be the survival function. Theorem 2 in Ciesielski and Taylor (1962) shows that for $n \geq 1$,

$$
\begin{aligned}
& S_{n}(t, \bar{y})=\sum_{k=1}^{\infty} \xi_{n, k} \exp \left(-\frac{q_{n, k}^{2}}{2 \bar{y}} \sigma^{2} t\right), \\
& \text { where } \quad \xi_{n, k}=\frac{1}{2^{\nu-1} \Gamma(\nu+1)} \frac{q_{n, k}^{\nu-1}}{J_{\nu+1}\left(q_{n, k}\right)},
\end{aligned}
$$

where $J_{\nu}(z)$ is the Bessel function of the first kind, where $\nu=(n-2) / 2$ and $q_{n, k}$ are the positive zeros of $J_{\nu}(z)$, indexed in ascending order according to $k$, and where $\Gamma$ is the gamma function. The hazard rate is then given by

$$
h(t, \bar{y})=-\frac{1}{S_{n}(t, \bar{y})} \frac{\partial S_{n}(t, \bar{y})}{\partial t} \quad \text { with asymptote } \lim _{t \rightarrow \infty} h(t, \bar{y})=\frac{q_{n, 1}^{2} \sigma^{2}}{2 \bar{y}} .
$$

For $n>2$, Hethcote (1970) provided the lower bound $q_{n, k}^{2}>\left(k-\frac{1}{4}\right)^{2} \pi^{2}+$ $\left(\frac{n}{2}-1\right)^{2}$.

Q.E.D.

PRoOf OF Proposition 6: We first establish the following lemma.

LEMMA 1: Let $z$ be distributed uniformly on the surface of the n-dimensional sphere of radius 1 . We use $x$ for the projection of $z$ in any of the dimensions, so $z_{i}=x \in[-1,1]$. The marginal distribution of $x=z_{i}$ has density

$$
\begin{aligned}
f_{n}(x) & =\int_{0}^{\infty} \frac{s^{(n-3) / 2} e^{-s / 2}}{2^{(n-1) / 2} \Gamma[(n-1) / 2]} \frac{e^{-s x^{2} /\left[2\left(1-x^{2}\right)\right]}}{\sqrt{2 \pi}} \frac{s^{1 / 2}}{\left(1-x^{2}\right)^{3 / 2}} d s \\
& =\frac{\Gamma(n / 2)}{\Gamma(1 / 2) \Gamma[(n-1) / 2]}\left(1-x^{2}\right)^{(n-3) / 2},
\end{aligned}
$$

where the $\Gamma$ function makes the density integrate to 1.

The lemma applies Theorem 2.1, part 1 in Song and Gupta (1997), using $p=2$ so that the norm is Euclidian and $k=1$ so that we have the marginal of one dimension. Now consider the case where the sphere has radius different from 1. Let $p \in \partial \mathcal{I}$. Then $p=\frac{p}{\sum_{i=1}^{n} p_{i}^{2}} \bar{y}=\frac{p}{\sqrt{\sum_{i=1}^{n} p_{i}^{2}}} \sqrt{\bar{y}}=z \sqrt{\bar{y}}$, where $z$ is uniformly distributed in the $n$-dimensional sphere of radius 1 . Thus each $p_{i}$ 
has the same distribution as $x \sqrt{\bar{y}}$. Using the change of variable formula, we obtain the required result. Some algebra using equation (13) for the density $w(\cdot)$ gives the expressions for the standard deviation, kurtosis and the other moments in the proposition.

For the convergence of $\Delta p_{i} / \operatorname{Std}\left(\Delta p_{i}\right)$ to a Normal, we show that $y=x^{2} n$ converges to a chi-squared distribution with 1 degree of freedom (d.o.f.), where $x$ is the marginal of a uniform distribution in the surface of the $n$ dimensional sphere. The p.d.f. of $y \in[0, n]$, the square of the standardized $x$, is $\frac{\Gamma(n / 2)}{n \Gamma((n-1) / 2) \Gamma(1 / 2)}\left(1-\left(\frac{y}{n}\right)\right)^{(n-3) / 2}\left(\frac{y}{n}\right)^{-1 / 2}$, and the p.d.f. of a chi-squared distribution with 1 d.o.f. is $\frac{\exp (-y / 2) y^{-1 / 2}}{\sqrt{2} \Gamma(1 / 2)}$. Then fixing $y$, taking logs in the ratio of the two p.d.f.'s, taking the limit as $n \rightarrow \infty$, and using that $\frac{\Gamma(n / 2) \sqrt{2}}{\Gamma((n-1) / 2) \sqrt{n}} \rightarrow 1$ as $n \rightarrow \infty$, we obtain the desired convergence result.

Q.E.D.

ProOf OF PROPOSITION 9: The forward Kolmogorov equation is

$$
0=\frac{1}{2} \frac{\partial^{2}}{\partial y^{2}}\left([2 \sigma \sqrt{y}]^{2} f(y)\right)-\frac{\partial}{\partial y}\left(n \sigma^{2} f(y)\right) \quad \text { for } \quad y \in(0, \bar{y}),
$$

with boundary conditions $1=\int_{0}^{\bar{y}} f(y) d y$ and $f(\bar{y})=0$. The first boundary ensures that $f$ is a density. The second is due to the fact that when the process hits $\bar{y}$, it is returned to the origin, so the mass escapes from this point. Equation (29) implies the second order ODE: $f^{\prime}(y)\left(\frac{n}{2}-2\right)=y f^{\prime \prime}(y)$. The solution of this ODE for $n \neq 2$ is $f(y)=A_{1} y^{n / 2-1}+A_{0}$ for two constants $A_{0}, A_{1}$ to be determined using the boundary conditions $0=A_{1}(\bar{y})^{n / 2-1}+A_{0}$ and $1=\frac{A_{1}}{n / 2}(\bar{y})^{n / 2}+A_{0} \bar{y}$. For $n=2$, the solution is $f(y)=-A_{1} \log (y)+A_{0}$ subject to the analogous boundary conditions. Solving for $A_{0}, A_{1}$ gives the desired expressions.

Q.E.D.

Proof of Proposition 10: The only result to be established is that the distribution of the sum of the coordinates of a vector uniformly distributed in the $n$-dimensional sphere has density given by equation (21). Using the result on page 387 of Khokhlov (2006), let $c: \mathbb{R} \rightarrow \mathbb{R}$ be measurable and let $L$ be the Lebesgue measure in an $n$-dimensional sphere. Then

$$
\begin{aligned}
& \int_{x \in \mathbb{R}^{n},\|x\|=1} c\left(x_{1}+\cdots+x_{n}\right) d L(x) \\
& =\frac{2 \pi^{(n-1) / 2}}{\Gamma\left(\frac{n-1}{2}\right)} \int_{-1}^{1} c(\sqrt{n} u)\left(1-u^{2}\right)^{(n-3) / 2} d u \\
& =\frac{2 \pi^{n / 2}}{\sqrt{n} \Gamma\left(\frac{1}{2}\right) \Gamma\left(\frac{n-1}{2}\right)} \int_{-\sqrt{n}}^{\sqrt{n}} c(\sqrt{n} u)\left(1-\left(\frac{\sqrt{n} u}{\sqrt{n}}\right)^{2}\right)^{(n-3) / 2} d(\sqrt{n} u) .
\end{aligned}
$$


Consider a function $c\left(x_{1}+\cdots+x_{n}\right)=1$ if $\alpha \leq x_{1}+\cdots+x_{n} \leq \beta$. Dividing by the surface area of the $n$-dimensional sphere, we obtain equation (21). Q.E.D.

ProOf OF PROPOSITION 11: We use expression (3.1) in Theorem 3.1 of Yin and Wang (2009) in equation (25) to obtain, for $n \geq 2$,

$$
\begin{aligned}
& \theta_{n}(\delta, t)=\sum_{m=0}^{\infty} \sum_{k=1}^{\infty} \varrho_{m, k}(\delta, \sqrt{\bar{y}}, n) \sigma^{2} e^{-\left(q_{m, k}^{2} /(2 n)\right)\left(n \sigma^{2} / \bar{y}\right) t}, \quad \text { where } \\
& \varrho_{m, k}(\delta, \sqrt{\bar{y}}, n)=\int_{\left\|p_{0}\right\|^{2} \leq \bar{y}}\left[\int_{\|p\|^{2}=\bar{y}} \frac{\left(p_{1}+p_{2}+\cdots+p_{n}\right)}{n}\right. \\
& \left.\times \varpi_{m, k}\left(p, p_{0}, \sqrt{\bar{y}}, n\right) d p\right] \lambda\left(p_{0}, \delta\right) d p_{0},
\end{aligned}
$$

where $\varpi_{m, k}$ are given by

$$
\begin{aligned}
\varpi_{m, k}\left(p, p_{0}, \sqrt{\bar{y}}, n\right)= & \left(\Gamma\left(\frac{n}{2}-1\right)\left(m+\frac{n}{2}-1\right) Z_{m}^{n / 2-1}\left(\cos \left(\angle p 0 p_{0}\right)\right)\right. \\
& \left.\times q_{m, k} J_{m+n / 2-1}\left(\frac{\|p\|}{\left\|p_{0}\right\|} q_{m, k}\right)\right) \\
& /\left(2 \pi^{n / 2}\|p\|^{n / 2+2}\left\|p_{0}\right\|^{n / 2-1} J_{m+n / 2-1}^{\prime}\left(q_{m, k}\right)\right),
\end{aligned}
$$

where $Z_{m}^{\nu}(x)$ denotes the Gegenbauer polynomials of degree $m$ and $\nu$, $\angle p 0 p_{0} \equiv\left(p \cdot p_{0}\right) /\left(\|p\|\left\|p_{0}\right\|\right)$ is the angle between $p_{0}$ and $p, q_{m, k}$ is the $k$ th (ordered) zero of the Bessel function $J_{m+n / 2-1}(\cdot)$, and $J_{m+n / 2-1}^{\prime}(\cdot)$ is the derivative of the Bessel function. The expression in Proposition 11 follows by integrating the right hand side of equation (30) with respect to $t$; thus, the coefficients $e_{m, k}$ are given by $e_{m, k}(\delta, \sqrt{\bar{y}}, n)=\varrho_{m, k}(\delta, \sqrt{\bar{y}}, n) \bar{y} 2 / q_{m, k}^{2}$. It is immediate that the homogeneity of degree 1 of $e_{m, k}(\cdot, n)$ is equivalent to the homogeneity of degree -1 of $\varrho_{m, k}(\cdot, n)$. To establish the homogeneity, we prove two properties: (i) Write $\lambda\left(p_{0}, \delta, \sqrt{\bar{y}}\right)$ that includes $\bar{y}$ as an argument, since it is an argument of $f$; see equation (19). Direct computation of equation (24) gives $\lambda\left(p_{0}, a \delta, a \sqrt{\bar{y}}\right)=\lambda\left(p_{0} / a, \delta, \sqrt{\bar{y}}\right) / a^{n}$ for any $a>0$. (ii) Direct computation of $\varpi_{m, k}$ reveals that the function satisfies: $\varpi_{m, k}\left(p, p_{0}, a \sqrt{\bar{y}}, n\right)=\varpi_{m, k}\left(p / a, p_{0} / a, \sqrt{\bar{y}}, n\right) / a^{n+1}$ for any $a>0$. Using (i) and (ii) in the expression for $\varrho_{m, k}$ and using the change of variables $p_{0}^{\prime}=p_{0} / a$ and $p^{\prime}=p / a$, and that the determinant of the Jacobian for the transformation. The determinant for the transformation of $p_{0}$ gives $a^{n}$ and the determinant for $p$ gives $a^{n-1}$ (the difference is because $\|p\|^{2}=\bar{y}$, so it is in $n-1$ dimensions). This proves the homogeneity of degree -1 of $\varrho_{m, k}$. 
Proof of Proposition 12: Let $\tilde{y} \equiv y / \bar{y}$ denote the values under the invariant distribution $f$ in equation (19), and let $\tilde{y}(\delta)$ denote the values of the same price gaps right after the monetary shock but before adjustment. Let $p$ be a vector of price gaps that satisfies $\tilde{y}=\|p\|^{2} / \bar{y}$ so that, for this $p$, $\tilde{y}(\delta)=\left\|p-\delta \mathbf{1}_{n}\right\|^{2} / \bar{y}$. Taking $y \in(0, \bar{y})$, developing the square in the expression for the corresponding value of $\tilde{y}(\delta)$, multiplying and dividing the cross-product term by $\sqrt{y}$, and using the definition of $\tilde{y}$ and $\operatorname{Std}\left(\Delta p_{i}\right)=\sqrt{\bar{y} / n}$, we have

$$
\begin{aligned}
\tilde{y}(\delta) & =\tilde{y}-2 \delta \sqrt{\frac{y}{\bar{y}}} \frac{\sum_{i=1}^{n} p_{i}}{\sqrt{y}} \frac{1}{\sqrt{\bar{y}}}+\frac{n}{\bar{y}} \delta^{2} \\
& =\tilde{y}-2 \delta \sqrt{\tilde{y}}\left(\frac{\sum_{i=1}^{n} p_{i}}{\sqrt{y}} \frac{1}{\sqrt{n}}\right) \frac{1}{\operatorname{Std}\left(\Delta p_{i}\right)}+\left(\frac{\delta}{\operatorname{Std}\left(\Delta p_{i}\right)}\right)^{2} .
\end{aligned}
$$

Conditional on $\tilde{y}$, we can regard $\tilde{y}(\delta)$ as a random variable, whose realizations correspond to each of the price gaps with $\|p\|^{2} / \bar{y}=\tilde{y}$, and where the price gaps $p$ are uniformly distributed on the sphere with square radius $y$. Proposition 10 gives the density of the random variable $\frac{\sum_{i=1}^{n} p_{i}}{\sqrt{y}}$, and using Proposition 6, it follows that for all $n$, its standard deviation is equal to 1 and its expected value is equal to 0 . Thus $\frac{\sum_{i=1}^{n} p_{i}}{\sqrt{y}} \frac{1}{\sqrt{n}}$ has an expected value equal to 0 and variance $1 / n$. Hence $\lim _{n \rightarrow \infty} \tilde{y}(\delta)=\tilde{y}+\left(\frac{\delta}{\operatorname{Std}\left(\Delta p_{i}\right)}\right)^{2}$, where the convergence to a (degenerate) random variable is in distribution. Combining this result for each $\tilde{y} \in[0,1]$ with Proposition 9 for $n \rightarrow \infty$, we obtain that the distribution of $\tilde{y}(\delta)$ converges to a uniform distribution in the interval $\left[\left(\frac{\delta}{\operatorname{Std}\left(\Delta p_{i}\right)}\right)^{2}, 1+\left(\frac{\delta}{\operatorname{Std}\left(\Delta p_{i}\right)}\right)^{2}\right]$. Immediately after the monetary shock, any firm with $y>\bar{y}$ or, equivalently, any firm with $\tilde{y}(\delta)>$ 1 , adjusts its prices. From here we see that the fraction of firms that adjust immediately after the shock, denoted by $\Phi_{n}$, converges to $\left(\delta / \operatorname{Std}\left(\Delta p_{i}\right)\right)^{2}$.

To characterize $\mathcal{P}_{n}$ for $t \geq 0$, we establish three properties: (i) the expected price change conditional on adjusting at time $t=0$ is equal to $\delta$, (ii) the fraction of firms that adjust for the first time after the shock between 0 and $t<\left[\delta-\Theta_{n}(\delta)\right] /\left[\delta N_{a}\right]$ equals $N_{a} t$, and (iii) the expected price change conditional on adjusting at time $0 \leq t<\left[\delta-\Theta_{n}(\delta)\right] /\left[\delta N_{a}\right]$ is equal to $\delta$. To establish (i), note that, as argued above, as $n \rightarrow \infty$, firms adjust their price if and only if they have a price gap $p$ before the monetary shock with square radius greater than $1-\left(\delta / \operatorname{Std}\left(\Delta p_{i}\right)\right)^{2}$. Since, in the invariant distribution, price gaps are uniformly distributed on each of the spheres, the expected price change across the firms with the same value of $y$ equals $\delta$. To establish (ii), note that, keeping constant $N_{a}$ as $n$ becomes large, the law of motion for $\tilde{y}$ in equation (12) converges to a deterministic one, namely $\tilde{y}_{t}=\tilde{y}_{0}+N_{a} t$. This, together with the 
uniform distribution for $\tilde{y}_{0}$, implies the desired result. Finally, (iii) follows from combining (i) and (ii).

Q.E.D.

\section{REFERENCES}

Alvarez, F., M. Gonzalez-RozadA, A. Neumeyer, And M. Beraja (2011): "From Hyperinflation to Stable Prices: Argentina's Evidence on Menu Cost Models," Manuscript, University of Chicago. [91]

Alvarez, F. E., AND F. Lippi (2012): "Price Setting With Menu Cost for Multi-Product Firms," NBER Working Papers 17923, National Bureau of Economic Research. [98,99,105,113,119],

(2014): "Supplement to 'Price Setting With Menu Cost for Multiproduct Firms'," Econometrica Supplemental Material, 82, http://www.econometricsociety.org/ecta/supmat/ 10662 miscellaneous.pdf; http://www.econometricsociety.org/ecta/supmat/10662_data_and_ programs.zip. [92]

Alvarez, F. E., H. Le Bihan, AND F. LiPPi (2013): "Small and Large Price Changes and the Propagation of Monetary Shocks,” Discussion Paper 9770, December, CEPR. [105,127]

Alvarez, F. E., F. LiPPI, AND L. PACIEllo (2011): “Optimal Price Setting With Observation and Menu Costs,” The Quarterly Journal of Economics, 126 (4), 1909-1960. [91,96]

ANDERSON, E., N. JAIMOVICH, AND D. SimeSTER (2012): "Price Stickiness: Empirical Evidence of the Menu Cost Channel," Working Paper, Duke. [105]

BARro, R. J. (1972): "A Theory of Monopolistic Price Adjustment," Review of Economic Studies, 39 (1), 17-26. [91,92,96,98,99,126]

Bertola, G., AND R. J. CABAllero (1994): “Irreversibility and Aggregate Investment,” Review of Economic Studies, 61 (2), 223-246. [115]

Bhattarai, S., AND R. SchOENLE (2011): "Multiproduct Firms and Price-Setting: Theory and Evidence From U.S. Producer Prices,” Working Paper 73, Globalization and Monetary Policy Institute, Federal Reserve Bank of Dallas. [102,104,105]

Bonomo, M., AND C. CARvalho (2004): "Endogenous Time-Dependent Rules and Inflation Inertia," Journal of Money, Credit and Banking, 36 (6), 1015-1041. [94,112]

Caballero, R. J. (1989): “Time Dependent Rules, Aggregate Stickiness and Information Externalities," Discussion Papers 198911, Columbia University. [94,112]

CABAllero, R. J., AND E. M. R. A. Engel (1991): "Dynamic (S, s) Economies,” Econometrica, 59 (6), 1659-1686. [93,108]

(1993): "Heterogeneity and Output Fluctuations in a Dynamic Menu-Cost Economy," Review of Economic Studies, 60 (1), 95-119. [90,93,108]

- (2007): "Price Stickiness in Ss Models: New Interpretations of Old Results," Journal of Monetary Economics, 54 (Supplement), 100-121. [90,93,108]

CAPlin, A., AND J. LEAHY (1997): "Aggregation and Optimization With State-Dependent Pricing," Econometrica, 65 (3), 601-626. [91,121]

Cavallo, A. (2010): "Scraped Data and Sticky Prices," Technical Report, MIT Sloan. [105]

Cavallo, A., AND R. Rigobon (2010): “The Distribution of the Size of Price Changes,” Working Paper, MIT. [126]

Chakrabarti, R., AND B. SCHOLNICK (2007): "The Mechanics of Price Adjustment: New Evidence on the (Un)Importance of Menu Costs," Managerial and Decision Economics, 28 (7), 657-668. [105]

Ciesielski, Z., AND S. J. TAYLOR (1962): "First Passage Times and Sojourn Times for Brownian Motion in Space and the Exact Hausdorff Measure of the Sample Path," Transactions of the American Mathematical Society, 103 (3), 434-450. [129]

DANZIGER, L. (1999): “A Dynamic Economy With Costly Price Adjustments," American Economic Review, 89 (4), 878-901. [91,107,121]

DiXIT, A. (1991): "Analytical Approximations in Models of Hysteresis," Review of Economic Studies, 58 (1), 141-151. [91,92,98,99,126] 
Dutta, S., M. Bergen, D. Levy, And R. Venable (1999): "Menu Costs, Posted Prices and Multiproduct Retailers," The Journal of Money, Credit and Banking, 31 (4), 683-703. [105]

EichenbaUm, M. S., N. JaImOVICH, S. Rebelo, AND J. SMith (2012): "How Frequent Are Small Price Changes?” NBER Working Paper 17956, National Bureau of Economic Research. [105]

FISHER, T. C. G., AND J. D. KONIECZNY (2000): "Synchronization of Price Changes by Multiproduct Firms: Evidence From Canadian Newspaper Prices,” Economics Letters, 68 (3), 271-277. [105]

Gagnon, E. (2009): "Price Setting During Low and High Inflation: Evidence From Mexico," Quarterly Journal of Economics, 124 (3), 1221-1263. [91]

GERTLER, M., AND J. LEAHY (2008): "A Phillips Curve With an Ss Foundation,” Journal of Political Economy, 116 (3), 533-572. [91,93]

GoldBerg, P., AND R. Hellerstein (2009): "How Rigid Are Producer Prices?” Working Papers 1184, Department of Economics, Center for Economic Policy Studies, Princeton University. [105]

Golosov, M., AND R. E. LuCAS, JR. (2007): “Menu Costs and Phillips Curves,” Journal of Political Economy, 115, 171-199. [89-91,93,94,104,106-108,121]

Hethсоте, H. W. (1970): "Bounds for Zeros of Some Special Functions," Proceedings of the American Mathematical Society, 25, 72-74. [129]

Karlin, S., AND H. M. TAYlor (1981): A Second Course in Stochastic Processes, Vol. 2. New York: Academic Press. [92,98,99]

KHOKHLOV, V. I. (2006): "The Uniform Distribution on a Sphere in $R^{S}$. Properties of Projections. I," Theory of Probability and Its Applications, 50, 386-399. [130]

KLENOW, P. J., AND B. A. MALIN (2010): "Microeconomic Evidence on Price-Setting," in Handbook of Monetary Economics, Vol. 3, ed. by B. M. Friedman and M. Woodford, Chapter 6. Amsterdam: Elsevier, 231-284. [89,90,105]

KolKIEwICZ, A. W. (2002): "Pricing and Hedging More General Double-Barrier Options," Journal of Computational Finance, 5 (3), 1-26. [115]

LACH, S., AND D. TsidDON (1996): "Staggering and Synchronization in Price-Setting: Evidence From Multiproduct Firms," American Economic Review, 86 (5), 1175-1196. [89,105]

(2007): "Small Price Changes and Menu Costs," Managerial and Decision Economics, 28 (7), 649-656. [89]

Levy, D., M. Bergen, S. DutTa, And R. Venable (1997): "The Magnitude of Menu Costs: Direct Evidence From Large US Supermarket Chains," The Quarterly Journal of Economics, 112 (3), 791-824. [105]

MidRigAN, V. (2009): “Menu Costs, Multi-Product Firms, and Aggregate Fluctuations,” Working Paper, NYU. [104,105,126]

(2011): "Menu Costs, Multi-Product Firms, and Aggregate Fluctuations," Econometrica, 79 (4), 1139-1180. [90,94,95,104,105]

ØKSEndal, B. K. (2000): Stochastic Differential Equations: An Introduction With Applications (Sixth Ed.). Berlin: Springer-Verlag. [98]

REIS, R. (2006): "Inattentive Producers," Review of Economic Studies, 73 (3), 793-821. [94,96, 112]

SHESHINSKI, E., AND Y. WeISS (1992): "Staggered and Synchronized Price Policies Under Inflation: The Multiproduct Monopoly Case," Review of Economic Studies, 59 (2), 331-359. [91]

SONG, D., AND A. K. GUPTA (1997): "Lp-Norm Uniform Distribution," Proceedings of the American Mathematical Society, 125 (2), 595-601. [103,129]

STOKEy, N. L. (2008): Economics of Inaction: Stochastic Control Models With Fixed Costs. Princeton: Princeton University Press. [91]

TAYLOR, J. B. (1980): “Aggregate Dynamics and Staggered Contracts," Journal of Political Economy, 88 (1), 1-23. [91,94,101,112]

TsidDon, D. (1993): "The (Mis)Behaviour of the Aggregate Price Level," Review of Economic Studies, 60 (4), 889-902. [91] 
Wendel, J. G. (1980): "Hitting Spheres With Brownian Motion," The Annals of Probability, 8 (1), 164-169. [120]

WULFSBERG, F. (2010): "Price Adjustments and Inflation-Evidence From Norwegian Consumer Price Data 1975-2004," Working Paper, Bank of Norway. [102,126]

YIN, C., AND C. WANG (2009): "Hitting Time and Place of Brownian Motion With Drift," The Open Statistics and Probability Journal, 1, 38-42. [120,131]

Dept. of Economics, University of Chicago, Rosenwald Hall, 1101 East 58th Street, Chicago, IL 60637, U.S.A.; f-alvarez1@uchicago.edu

and

EIEF and University of Sassari, via Sallustiana, 62, 00187 Rome, Italy; flippi@uniss.it.

Manuscript received March, 2012; final revision received June, 2013. 